\title{
Endpoint regularity of discrete multilinear fractional nontangential maximal functions
}

Daiqing Zhang ${ }^{1 *}$

${ }^{*}$ Correspondence: zhangdaiqing2011@163.com ${ }^{1}$ College of Mathematics and Physics, Fujian University of Technology, Fuzhou, China

\section{Springer}

\begin{abstract}
Given $m \geq 1,0 \leq \lambda \leq 1$, and a discrete vector-valued function $\vec{f}=\left(f_{1}, \ldots, f_{m}\right)$ with each $f_{j}: \mathbb{Z}^{d} \rightarrow \mathbb{R}$, we consider the discrete multilinear fractional nontangential maximal operator

$$
\mathrm{M}_{\alpha, \mathcal{B}}^{\lambda}(\vec{f})(\vec{n})=\sup _{\substack{r>0, \vec{x} \in \mathbb{R}^{d} \\|\vec{n}-\vec{x}| \leq \lambda r}} \frac{1}{N\left(B_{r}(\vec{x})\right)^{m-\frac{\alpha}{d}}} \prod_{j=1}^{m} \sum_{\vec{k} \in B_{r}(\vec{x}) \cap \mathbb{Z}^{d}}\left|f_{j}(\vec{k})\right|,
$$

where $\mathcal{B}$ is the collection of all open balls $B \subset \mathbb{R}^{d}, B_{r}(\vec{x})$ is the open ball in $\mathbb{R}^{d}$ centered at $\vec{x} \in \mathbb{R}^{d}$ with radius $r$, and $N\left(B_{r}(\vec{x})\right)$ is the number of lattice points in the set $B_{r}(\vec{x})$. We show that the operator $\vec{f} \mapsto\left|\nabla \mathrm{M}_{\alpha, \mathcal{B}}^{\lambda}(\vec{f})\right|$ is bounded and continuous from $\ell^{1}\left(\mathbb{Z}^{d}\right) \times \ell^{1}\left(\mathbb{Z}^{d}\right) \times \cdots \times \ell^{1}\left(\mathbb{Z}^{d}\right)$ to $\ell^{q}\left(\mathbb{Z}^{d}\right)$ if $0 \leq \alpha<m d$ and $q \geq 1$ such that $q>\frac{d}{m d-\alpha+1}$. We also prove that the same result also holds for the discrete multilinear fractional nontangential maximal operators associated with cubes. These results we obtained represent significant and natural extensions of what was known previously.
\end{abstract}

Keywords: Discrete multilinear fractional nontangential maximal operator; Discrete multilinear fractional maximal operator; Bounded variation; Continuity

\section{Introduction}

The regularity theory of maximal operators has been the subject of many recent articles in harmonic analysis. The first work was due to Kinnunen [12] who observed that the centered Hardy-Littlewood maximal operator $\mathcal{M}$ is bounded on the first order Sobolev space $W^{1, p}\left(\mathbb{R}^{d}\right)$ for $1<p \leq \infty$. Since then, the regularity properties of the various kinds of maximal operators have been studied by many authors. See $[8,11,13-16,19,20,24,25,28$, 30 , 31] for example. Since $\mathcal{M}: L^{1}\left(\mathbb{R}^{d}\right) \rightarrow L^{1}\left(\mathbb{R}^{d}\right)$ is not bounded, the endpoint regularity of maximal operators seems to be a deeper issue. A crucial question was posed by Hajłasz and Onninen in [11].

Problem $1.1([11])$ Is the operator $f \mapsto|\nabla \mathcal{M} f|$ bounded from $W^{1,1}\left(\mathbb{R}^{d}\right)$ to $L^{1}\left(\mathbb{R}^{d}\right)$ ?

In 2002, Tanaka [35] first proved that the uncentered Hardy-Littlewood maximal function $\widetilde{\mathcal{M}} f$ is weakly differentiable and satisfies

$$
\left\|(\widetilde{\mathcal{M}} f)^{\prime}\right\|_{L^{1}(\mathbb{R})} \leq 2\left\|f^{\prime}\right\|_{L^{1}(\mathbb{R})}
$$

(c) The Author(s) 2019. This article is distributed under the terms of the Creative Commons Attribution 4.0 International License (http://creativecommons.org/licenses/by/4.0/), which permits unrestricted use, distribution, and reproduction in any medium, provided you give appropriate credit to the original author(s) and the source, provide a link to the Creative Commons license, and indicate if changes were made. 
if $f \in W^{1,1}(\mathbb{R})$. Later on, Tanaka's result was refined by Aldaz and Pérez Lázaro [1] who showed that if $f$ is of bounded variation on $\mathbb{R}$, then $\widetilde{\mathcal{M}} f$ is absolutely continuous and

$$
\operatorname{Var}(\widetilde{\mathcal{M}} f) \leq \operatorname{Var}(f)
$$

where $\operatorname{Var}(f)$ denotes the total variation of $f$. The above result directly implies that

$$
\left\|(\widetilde{\mathcal{M}} f)^{\prime}\right\|_{L^{1}(\mathbb{R})} \leq\left\|f^{\prime}\right\|_{L^{1}(\mathbb{R})}
$$

if $f \in W^{1,1}(\mathbb{R})$ (see also [23] for a new proof). Notice that inequalities (2) and (3) are sharp. In the centered case, Kurka [17] showed that if $f$ is of bounded variation on $\mathbb{R}$, then

$$
\operatorname{Var}(\mathcal{M} f) \leq 240,004 \operatorname{Var}(f)
$$

It was also shown in [17] that if $f \in W^{1,1}(\mathbb{R})$, then $\mathcal{M} f$ is weakly differentiable and inequality (3) also holds for $\mathcal{M}$ with constant $C=240,004$. It is currently unknown whether inequalities (2) and (3) also hold for $\mathcal{M}$. Recently, Carneiro and Madrid [7] extended inequalities (2) and (3) to a fractional setting. Very recently, we [26] extended the result of [7] to a multisublinear setting. Other interesting works related to this theory are $[4,9,10$, 22, 33].

On the other hand, the investigation of the regularity of discrete maximal operators has also attracted the attention of many authors (cf. [2, 5, 7, 18, 21, 24, 27, 29, 32, 36, 37]). Let us recall some definitions and background. For $d \geq 1$ and $\vec{n}=\left(n_{1}, n_{2}, \ldots, n_{d}\right) \in \mathbb{Z}^{d}$, we set $|\vec{n}|=\left(\sum_{i=1}^{d}\left|n_{i}\right|^{2}\right)^{1 / 2}$ and $\|\vec{n}\|_{\infty}=\sup _{1 \leq i \leq d}\left|n_{i}\right|$. For a discrete function $f: \mathbb{Z}^{d} \rightarrow \mathbb{R}$ and $1 \leq p \leq \infty$, we define its $\ell^{p}\left(\mathbb{Z}^{d}\right)$-norm by $\|f\|_{\ell^{p}\left(\mathbb{Z}^{d}\right)}=\left(\sum_{\vec{n} \in \mathbb{Z}^{d}}|f(\vec{n})|^{p}\right)^{1 / p}$ if $1 \leq p<\infty$ and $\|f\|_{\ell \infty\left(\mathbb{Z}^{d}\right)}=\sup _{\vec{n} \in \mathbb{Z}^{d}}|f(\vec{n})|$. Formally, define the discrete analogue of the Sobolev spaces by

$$
W^{1, p}\left(\mathbb{Z}^{d}\right):=\left\{f: \mathbb{Z}^{d} \rightarrow \mathbb{R} \mid\|f\|_{1, p}=\|f\|_{\ell^{p}\left(\mathbb{Z}^{d}\right)}+\|\nabla f\|_{\ell^{p}\left(\mathbb{Z}^{d}\right)}<\infty\right\}
$$

where $\nabla f$ is the gradient of a discrete function $f$ and is defined by

$$
\nabla f(\vec{n})=\left(D_{1} f(\vec{n}), D_{2} f(\vec{n}), \ldots, D_{d} f(\vec{n})\right)
$$

and $D_{l} f(\vec{n})$ is the partial derivative of $f$ denoted by

$$
D_{l} f(\vec{n})=f\left(\vec{n}+\vec{e}_{l}\right)-f(\vec{n})
$$

and $\vec{e}_{l}=(0, \ldots, 0,1,0, \ldots, 0)$ is the canonical $l$ th base vector, $l=1,2, \ldots, d$. Observe that

$$
\|f\|_{\ell p}\left(\mathbb{Z}^{d}\right) \leq\|f\|_{1, p} \leq(2 d+1)\|f\|_{\ell^{p}\left(\mathbb{Z}^{d}\right)} \quad \forall 1 \leq p \leq \infty
$$

It follows that

$$
W^{1, p}\left(\mathbb{Z}^{d}\right)=\ell^{p}\left(\mathbb{R}^{d}\right) \quad \forall 1 \leq p \leq \infty .
$$


We denote by $\operatorname{BV}\left(\mathbb{Z}^{d}\right)$ the set of all functions of bounded variation defined on $\mathbb{Z}^{d}$, where the total variation of $f: \mathbb{Z}^{d} \rightarrow \mathbb{R}$ is defined by

$$
\operatorname{Var}(f)=\sum_{l=1}^{d}\left\|D_{l} f\right\|_{\ell^{1}\left(\mathbb{Z}^{d}\right)} .
$$

It is clear that

$$
\begin{aligned}
& \|\nabla f\|_{\ell^{1}\left(\mathbb{Z}^{d}\right)} \leq \operatorname{Var}(f) \leq d\|\nabla f\|_{\ell^{1}\left(\mathbb{Z}^{d}\right)}, \\
& W^{1,1}\left(\mathbb{R}^{d}\right)=\ell^{1}\left(\mathbb{Z}^{d}\right) \subsetneq \operatorname{BV}\left(\mathbb{Z}^{d}\right) .
\end{aligned}
$$

Let $m \geq 1$ and $0 \leq \alpha<m d$. For a vector-valued function $\vec{f}=\left(f_{1}, \ldots, f_{m}\right)$ with each $f_{j}$ : $\mathbb{Z}^{d} \rightarrow \mathbb{R}$ being a discrete function, we define the discrete centered $m$-sublinear fractional maximal operator $\mathrm{M}_{\alpha}$ by

$$
\mathrm{M}_{\alpha}(\vec{f})(\vec{n})=\sup _{r>0} \frac{1}{N\left(B_{r}(\vec{n})\right)^{m-\frac{\alpha}{d}}} \prod_{j=1}^{m} \sum_{\vec{k} \in B_{r}(\vec{n}) \cap \mathbb{Z}^{d}}\left|f_{j}(\vec{k})\right|,
$$

where $B_{r}(\vec{n})$ is the open ball in $\mathbb{R}^{d}$ centered at $\vec{n}$ with radius $r$ and $N\left(B_{r}(\vec{n})\right)$ is the number of lattice points in the set $B_{r}(\vec{n})$. The uncentered version of $\mathrm{M}_{\alpha}$ is given by

$$
\tilde{\mathrm{M}}_{\alpha}(\vec{f})(\vec{n})=\sup _{r>0, \vec{n} \in B_{r}} \frac{1}{N\left(B_{r}\right)^{m-\frac{\alpha}{d}}} \prod_{j=1}^{m} \sum_{\vec{k} \in B_{r} \cap \mathbb{Z}^{d}}\left|f_{j}(\vec{k})\right|,
$$

where the supremum is taken over all open balls $B_{r}$ in $\mathbb{R}^{d}$ containing the point $\vec{n}$ with radius $r$. When $m=1$, the operator $\mathrm{M}_{\alpha}$ (resp., $\widetilde{\mathrm{M}}_{\alpha}$ ) reduces to the discrete centered (resp., uncentered) fractional maximal operator $M_{\alpha}$ (resp., $\widetilde{M}_{\alpha}$ ). Particularly, when $\alpha=0$, the operator $M_{\alpha}$ (resp., $\left.\widetilde{M}_{\alpha}\right)$ is just the discrete centered (resp., uncentered) Hardy-Littlewood maximal operator $M$ (resp., $\widetilde{M}$ ).

The study of the regularity properties of discrete maximal operators was initiated by Bober et al. [2] in 2012 when they observed that

$$
\operatorname{Var}(\tilde{M} f) \leq \operatorname{Var}(f)
$$

and

$$
\operatorname{Var}(M f) \leq\left(2+\frac{146}{315}\right)\|f\|_{\ell^{1}(\mathbb{Z})}
$$

It was noticed that inequality (8) is sharp and (9) is not sharp. Inequality (9) with the sharp constant $C=2$ was proved by Madrid in [32] (see [32, Theorem 1]). It was known that inequality (8) for $M$ was established by Temur in [36] (with constant $C=294,912,004$ ). It is unknown whether inequality (8) also holds for M. Recently, Carneiro and Madrid [7] and Liu [18] extended (8) and [32, Theorem 1] to the fractional setting, respectively. More recently, in the remarkable paper [6], Carneiro et al. established the $\mathrm{BV}(\mathbb{Z})$-continuity of the discrete centered and uncentered Hardy-Littlewood maximal operator. For general 
dimension $d \geq 1$, Carneiro and Hughes [5] showed that both $M$ and $\widetilde{M}$ map $\ell^{1}\left(\mathbb{Z}^{d}\right)$ into $\mathrm{BV}\left(\mathbb{Z}^{d}\right)$ boundedly and continuously. Recently, Carneiro and Madrid [7] extended the result of [5] to a fractional setting. Very recently, we [27] extended the result of [5] to a multisublinear fractional setting.

Let us recall the main result of [27], which can be stated as follows.

Theorem 1.2 ([27]) Let $0 \leq \alpha<(m-1) d+1$. Then both $\mathrm{M}_{\alpha}$ and $\tilde{\mathrm{M}}_{\alpha}$ map $\ell^{1}\left(\mathbb{Z}^{d}\right) \times \ell^{1}\left(\mathbb{Z}^{d}\right) \times$ $\cdots \times \ell^{1}\left(\mathbb{Z}^{d}\right)$ into $\mathrm{BV}\left(\mathbb{Z}^{d}\right)$ boundedly and continuously.

The aim of this paper is to investigate the endpoint regularity of the discrete multilinear fractional nontangential maximal operator associated with balls or cubes.

Definition 1.3 Let $0 \leq \alpha<m d$ and $0 \leq \lambda \leq 1$. For a vector-valued function $\vec{f}=\left(f_{1}, \ldots, f_{m}\right)$ with each $f_{j}: \mathbb{Z}^{d} \rightarrow \mathbb{R}$ being a discrete function, we define the discrete multilinear fractional nontangential maximal operator associated with balls $\mathrm{M}_{\alpha, \mathcal{B}}^{\gamma}$ by

$$
\mathrm{M}_{\alpha, \mathcal{B}}^{\gamma}(\vec{f})(\vec{n})=\sup _{\substack{r>0, \vec{x} \in \mathbb{R}^{d} \\|\vec{n}-\vec{x}| \leq \lambda r}} \frac{1}{N\left(B_{r}(\vec{x})\right)^{m-\frac{\alpha}{d}}} \prod_{j=1}^{m} \sum_{\vec{k} \in B_{r}(\vec{x}) \cap \mathbb{Z}^{d}}\left|f_{j}(\vec{k})\right|,
$$

where $\mathcal{B}$ is the collection of all open balls $B \subset \mathbb{R}^{d}$. The discrete multilinear fractional nontangential maximal operator associated with cubes $\mathrm{M}_{\alpha, \mathcal{R}}^{\lambda}$ is defined by

$$
\mathrm{M}_{\alpha, \mathcal{R}}^{\lambda}(\vec{f})(\vec{n})=\sup _{\substack{r>0, \vec{x} \in \mathbb{R}^{d} \\\|\vec{n}-\vec{x}\|}} \frac{1}{N\left(R_{r}(\vec{x})\right)^{m-\frac{\alpha}{d}}} \prod_{j=1}^{m} \sum_{\vec{k}, R_{r}(\vec{x}) \in \mathcal{R}} \sum_{\vec{k} \in R_{r}(\vec{x}) \cap \mathbb{Z}^{d}}\left|f_{j}(\vec{k})\right|,
$$

where $\mathcal{R}$ is the collection of all open cubes $R \subset \mathbb{R}^{d}$ with sides parallel to the coordinate axes and $R_{r}(\vec{x})$ is the open cube in $\mathbb{R}^{d}$ centered at $\vec{x}$ with length of side $2 r$.

One can easily check that

$$
\begin{aligned}
& \mathrm{M}_{\alpha}(\vec{f})(\vec{n})=\mathrm{M}_{\alpha, \mathcal{B}}^{0}(\vec{f})(\vec{n}) \leq \mathrm{M}_{\alpha, \mathcal{B}}^{\lambda}(\vec{f})(\vec{n}) \leq \mathrm{M}_{\alpha, \mathcal{B}}^{1}(\vec{f})(\vec{n})=\tilde{\mathrm{M}}_{\alpha}(\vec{f})(\vec{n}) \quad \forall \vec{n} \in \mathbb{Z}^{d} ; \\
& \mathrm{M}_{\alpha, \mathcal{B}}^{\lambda}(\vec{f})(\vec{n}) \sim_{\alpha, m, d, \lambda} \mathrm{M}_{\alpha, \mathcal{R}}^{\lambda}(\vec{f})(\vec{n}) \quad \forall \vec{n} \in \mathbb{Z}^{d} .
\end{aligned}
$$

By relationships (10)-(11) and the bounds for $\widetilde{\mathrm{M}}_{\alpha}$, we obtain

$$
\left\|\mathrm{M}_{\alpha, \mathcal{R}}^{\lambda}(\vec{f})\right\|_{\ell^{q}\left(\mathbb{Z}^{d}\right)} \lesssim_{\alpha, m, d, \lambda}\left\|\mathrm{M}_{\alpha, \mathcal{B}}^{\lambda}(\vec{f})\right\|_{\ell^{q}\left(\mathbb{Z}^{d}\right)} \lesssim_{\alpha, m, d, p_{1}, \ldots, p_{d}, q} \prod_{j=1}^{m}\left\|f_{j}\right\|_{\ell^{p_{j}}\left(\mathbb{Z}^{d}\right)},
$$

if $1<p_{i} \leq \infty(i=1, \ldots, d), 1 \leq q \leq \infty$ for $\alpha=0$, and $1<p_{i}<\infty(i=1, \ldots, d), 1 \leq q<\infty$ for $0<\alpha<m d$ and $\frac{1}{q} \leq \frac{1}{p_{1}}+\cdots+\frac{1}{p_{m}}-\frac{\alpha}{d}$. One can easily check that

$$
\left|\mathrm{M}_{\alpha, \mathcal{B}}^{\lambda}(\vec{f})(\vec{n})-\mathrm{M}_{\alpha, \mathcal{B}}^{\lambda}(\vec{g})(\vec{n})\right| \leq \sum_{j=1}^{m} \mathrm{M}_{\alpha, \mathcal{B}}^{\lambda}\left(\vec{F}_{j}\right)(\vec{n}) \quad \forall \vec{n} \in \mathbb{Z}^{d}
$$

where $\vec{f}=\left(f_{1}, \ldots, f_{m}\right), \vec{g}=\left(g_{1}, \ldots, g_{m}\right)$ and $\vec{F}_{j}=\left(f_{1}, \ldots, f_{j-1}, f_{j}-g_{j}, g_{j+1}, \ldots, g_{m}\right)$. It follows from (4) and (12)-(13) that both $\mathrm{M}_{\alpha, \mathcal{B}}^{\lambda}$ and $\mathrm{M}_{\alpha, \mathcal{R}}^{\lambda}$ are bounded and continuous from $W^{1, p_{1}}\left(\mathbb{Z}^{d}\right) \times$ 
$W^{1, p_{2}}\left(\mathbb{Z}^{d}\right) \times \cdots \times W^{1, p_{m}}\left(\mathbb{Z}^{d}\right) \rightarrow W^{1, q}\left(\mathbb{Z}^{d}\right)$ provided that $1<p_{i} \leq \infty(i=1, \ldots, m), 1 \leq q \leq$ $\infty$ for $\alpha=0$, and $1<p_{i} \leq \infty(i=1, \ldots, m), 1 \leq q<\infty$ for $0<\alpha<m d$, and $\frac{1}{q} \leq \frac{1}{p_{1}}+\cdots+$ $\frac{1}{p_{m}}-\frac{\alpha}{d}$. In addition, it is clear that both $\mathrm{M}_{\alpha, \mathcal{B}}^{\lambda}$ and $\mathrm{M}_{\alpha, \mathcal{R}}^{\lambda}$ are not bounded from $\ell^{1}\left(\mathbb{Z}^{d}\right) \times$ $\ell^{1}\left(\mathbb{Z}^{d}\right) \times \cdots \times \ell^{1}\left(\mathbb{Z}^{d}\right)$ to $\ell^{1}\left(\mathbb{Z}^{d}\right)$.

Based on the above bounds for $\mathrm{M}_{\alpha, \mathcal{B}}^{\lambda}$ and $\mathrm{M}_{\alpha, \mathcal{R}}^{\lambda}$, Theorem 1.2, (7), and (10)-(11), a question that arises naturally is the following.

Problem 1.4 Are both the operators $\mathrm{M}_{\alpha, \mathcal{B}}^{\lambda}$ and $\mathrm{M}_{\alpha, \mathcal{R}}^{\lambda}$ bounded and continuous from $\ell^{1}\left(\mathbb{Z}^{d}\right) \times \ell^{1}\left(\mathbb{Z}^{d}\right) \times \cdots \times \ell^{1}\left(\mathbb{Z}^{d}\right)$ to $\mathrm{BV}\left(\mathbb{Z}^{d}\right)$ ?

We would like to point out that Problem 1.4 seems to be affirmative and expected. However, we will present a positive answer to Problem 1.4 by the following more general conclusion.

Theorem 1.5 Let $0 \leq \alpha<m d, 0 \leq \lambda \leq 1$, and $0 \leq \beta \leq 1$. Let $q \geq 1$ such that $q>\frac{d}{m d-\alpha+\beta}$. Then the operator $\vec{f} \mapsto\left|\nabla \mathrm{M}_{\alpha, \mathcal{B}}^{\lambda}(\vec{f})\right|$ is bounded and continuous from $\ell^{1}\left(\mathbb{Z}^{d}\right) \times \ell^{1}\left(\mathbb{Z}^{d}\right) \times \cdots \times$ $\ell^{1}\left(\mathbb{Z}^{d}\right)$ to $\ell^{q}\left(\mathbb{Z}^{d}\right)$. Moreover,

$$
\left\|\nabla \mathrm{M}_{\alpha, \mathcal{B}}^{\lambda}(\vec{f})\right\|_{\ell^{q}\left(\mathbb{Z}^{d}\right)} \lesssim_{\alpha, m, d, q, \beta, \lambda} \sum_{\mu=1}^{m}\left(\left\|f_{\mu}\right\|_{\ell^{1}\left(\mathbb{Z}^{d}\right)}^{\beta}\left\|\nabla f_{\mu}\right\|_{\ell^{1}\left(\mathbb{Z}^{d}\right)}^{1-\beta}\right) \prod_{1 \leq j \neq \mu \leq m}\left\|f_{j}\right\|_{\ell^{1}\left(\mathbb{Z}^{d}\right)}
$$

for all $\vec{f}=\left(f_{1}, \ldots, f_{m}\right)$ with each $f_{j} \in \ell^{1}\left(\mathbb{Z}^{d}\right)$. The same results hold for the operator $\mathrm{M}_{\alpha, \mathcal{R}}^{\lambda}$.

Remark 1.6 It should be pointed out that inequality (14) holds only if $q \geq \frac{d}{m d-\alpha+\beta}$. To see this, let $l$ be an integer such that $l \geq 8 \sqrt{d}$. Taking $f_{j}(\vec{n})=\chi_{\{|\vec{n}| \leq l\}}(\vec{n})$ for all $1 \leq j \leq m$, one can verify that $\left\|f_{j}\right\|_{\ell^{1}\left(\mathbb{Z}^{d}\right)} \sim_{d} l^{d},\left\|\nabla f_{j}\right\|_{\ell^{1}\left(\mathbb{Z}^{d}\right)} \sim_{d} l^{d-1}$ and $\left\|\nabla \mathrm{M}_{\alpha, \mathcal{B}}^{\lambda}(\vec{f})\right\|_{\ell q\left(\mathbb{Z}^{d}\right)} \gtrsim_{d^{q^{q}+\alpha-1}}$. It follows that

$$
\frac{\left\|\nabla \mathrm{M}_{\alpha, \mathcal{B}}^{\lambda}(\vec{f})\right\|_{\ell^{q}\left(\mathbb{Z}^{d}\right)}}{\sum_{\mu=1}^{m}\left(\left\|f_{\mu}\right\|_{\ell^{1}\left(\mathbb{Z}^{d}\right)}^{\beta}\left\|\nabla f_{\mu}\right\|_{\ell^{1}\left(\mathbb{Z}^{d}\right)}^{1-\beta}\right) \prod_{1 \leq j \neq \mu \leq m}\left\|f_{j}\right\|_{\ell^{1}\left(\mathbb{Z}^{d}\right)}} \gtrsim_{\alpha, m, d, \beta, q} l^{\frac{d}{q}+\alpha-m d-\beta} .
$$

This yields our claim by letting $l \rightarrow \infty$.

As several direct corollaries of Theorem 1.5, we obtain the following.

Corollary 1.7 Let $m \geq 1,0 \leq \lambda \leq 1$, and $0 \leq \alpha<(m-1) d+1$. Then both $\mathrm{M}_{\alpha, \mathcal{B}}^{\lambda}$ and $\mathrm{M}_{\alpha, \mathcal{R}}^{\lambda}$ are bounded and continuous from $\ell^{1}\left(\mathbb{Z}^{d}\right) \times \ell^{1}\left(\mathbb{Z}^{d}\right) \times \cdots \times \ell^{1}\left(\mathbb{Z}^{d}\right)$ to $\mathrm{BV}\left(\mathbb{Z}^{d}\right)$.

Corollary 1.8 Let $m>1,0 \leq \lambda \leq 1$, and $0 \leq \alpha<(m-1)$ d. Then both $\mathrm{M}_{\alpha, \mathcal{B}}^{\lambda}$ and $\mathrm{M}_{\alpha, \mathcal{R}}^{\lambda}$ are bounded from $\mathrm{BV}\left(\mathbb{Z}^{d}\right) \times \ell^{1}\left(\mathbb{Z}^{d}\right) \times \cdots \times \ell^{1}\left(\mathbb{Z}^{d}\right)$ to $\mathrm{BV}\left(\mathbb{Z}^{d}\right)$.

Remark 1.9 When $\beta=1$ (resp., $\beta=0)$ and $0 \leq \alpha<(m-1) d+1$ (resp., $0 \leq \alpha<(m-1) d$ ), then $\frac{d}{m d-\alpha+\beta}<1$. Thus Theorem 1.5 yields Corollaries 1.7 and 1.8. On the other hand, Corollary 1.7 extends Theorem B, which corresponds to the case $\lambda=0$ and $\lambda=1$.

Remark 1.10 Our main results are new even in the special case $m=1$ and $\alpha=0$. 
The paper is organized as follows. Section 2 contains some preliminary notation and a useful subtle summability lemma. The proof of Theorem 1.5 will be given in Sect. 3. We would like to remark that our arguments are motivated by [7], but our methods and techniques are more simple and direct than those of [7]. It is worth mentioning that there are three virtues: (i) In the previous papers [5, 7, 27], the authors established the endpoint regularities of the discrete maximal operator and its fractional version and multilinear fractional version by dealing with their centered case and uncentered case individually. Here, we give a uniform handling method of proving the regularity properties of discrete centered and uncentered maximal operators. (ii) In the precise papers [5, 7, 27], the proofs of the corresponding continuity results are highly dependent on the Brezis-Lieb lemma [3]. Moreover, the discrete versions of Luiro's lemma (see [5, Lemmas 3-4] and [7, Lemmas 4-5] played key roles in the proofs of the corresponding continuity results in [5, 7]. However, these useful lemmas are unnecessary in our proofs. (iii) Although our main result greatly improves the main result of [27], our methods and techniques are more simple than those of [27].

Throughout this paper, if there exists a constant $c>0$ depending only on $\vartheta$ such that $A \leq c B$, we then write $A \lesssim_{\vartheta} B$ or $B{ }_{\vartheta} A$; and if $A \lesssim_{\vartheta} B \lesssim_{\vartheta} A$, we then write $A \sim_{\vartheta} B$. In what follows, for a set $E \subset \mathbb{R}^{d}$, we set $E^{c}=\left\{x \in \mathbb{R}^{d} ; x \notin E\right\}$. We shall use the conventions $\prod_{i \in \emptyset} a_{i}=1$ and $\sum_{i \in \emptyset} a_{i}=0$.

\section{Preliminaries}

We start by presenting some preliminary notation. It was shown in [34] that

$$
c_{d}(r-\sqrt{d} / 2)^{d} \leq N\left(B_{r}(\vec{n})\right) \leq c_{d}(r+\sqrt{d} / 2)^{d} \quad \forall \vec{n} \in \mathbb{Z}^{d} \text { and } r>\sqrt{d} / 2,
$$

where $c_{d}=\frac{2 d^{d / 2}}{\Gamma(d / 2) d}$. It is clear that

$$
\chi_{\{0<r \leq 1\}}(r)+(2[r-1]+1)^{d} \chi_{\{r>1\}}(r) \leq N\left(R_{r}(\vec{n})\right) \leq(2[r]+1)^{d} \quad \forall r>0 \text { and } \vec{n} \in \mathbb{Z}^{d} \text {. }
$$

Here $[x]=\{k \in \mathbb{Z} ; k \leq x\}$. Fix $\vec{x} \in \mathbb{R}^{d} \backslash \mathbb{Z}^{d}$, there exist two lattice points $\vec{n}_{1} \in \mathbb{Z}^{d}$ and $\overrightarrow{n_{2}} \in \mathbb{Z}^{d}$ such that $\left|\overrightarrow{n_{1}}-\vec{x}\right| \leq \sqrt{d} / 2$ and $\left\|\overrightarrow{n_{2}}-\vec{x}\right\|_{\infty} \leq 1 / 2$ and

$$
\begin{aligned}
& B_{r-\sqrt{d} / 2}\left(\vec{n}_{1}\right) \subset B_{r}(\vec{x}) \subset B_{r+\sqrt{d} / 2}\left(\vec{n}_{1}\right) \quad \forall r>\sqrt{d} / 2 ; \\
& R_{r-1 / 2}\left(\vec{n}_{2}\right) \subset R_{r}(\vec{x}) \subset R_{r+1 / 2}\left(\vec{n}_{2}\right) \quad \forall r>1 / 2 .
\end{aligned}
$$

Consequently,

$$
\begin{aligned}
& c_{d}(r-\sqrt{d})^{d} \leq N\left(B_{r}(\vec{x})\right) \leq c_{d}(r+\sqrt{d})^{d} \quad \forall x \in \mathbb{R}^{d} \text { and } r>\sqrt{d} ; \\
& (2[r-3 / 2]+1)^{d} \leq N\left(R_{r}(\vec{x})\right) \leq(2[r+1 / 2]+1)^{d} \quad \forall x \in \mathbb{R}^{d} \text { and } r>3 / 2 .
\end{aligned}
$$

It follows from (18) that

$$
2^{d}(r-2)^{d} \leq N\left(R_{r}(\vec{x})\right) \leq 2^{d}(r+1)^{d} \quad \forall x \in \mathbb{R}^{d} \text { and } r \geq 5 / 2 .
$$

Define the functions $F(r)$ and $G(r)$ on $(0, \infty)$ by

$$
F(r)=c_{d}(r-\sqrt{d})^{d} \chi_{\{r \geq 4 \sqrt{d}\}}(r)+\chi_{\{0<r<4 \sqrt{d}\}}(r) ;
$$




$$
G(r)=2^{d}(r-2)^{d} \chi_{\{r \geq 4\}}(r)+\chi_{\{0<r<4\}}(r) .
$$

Observe from (17) and (19) that

$$
N\left(B_{r}(\vec{x})\right) \geq F(r) \quad \text { and } \quad N\left(R_{r}(\vec{x})\right) \geq G(r) \quad \forall \vec{x} \in \mathbb{R}^{d} \text { and } r>0 .
$$

We can claim that

$$
N\left(B_{r+1}(\vec{x})\right)^{\gamma}-N\left(B_{r}(\vec{x})\right)^{\gamma} \lesssim_{\gamma, d} F(r)^{\gamma-1 / d} \quad \forall r>0, \gamma \geq 1 \text { and } \vec{x} \in \mathbb{R}^{d}
$$

To see this, fix $\vec{x} \in \mathbb{R}^{d}$, when $r \geq 4 \sqrt{d}$, by (17) and the differential mean value theorem,

$$
N\left(B_{r+1}(\vec{x})\right)^{\gamma}-N\left(B_{r}(\vec{x})\right)^{\gamma} \leq c_{d}^{\gamma}\left((r+1+\sqrt{d})^{\gamma d}-(r-\sqrt{d})^{\gamma d}\right) \lesssim_{\gamma, d}(r-\sqrt{d})^{\gamma d-1} .
$$

When $0<r<4 \sqrt{d}$, we get from (15) that

$$
N\left(B_{r+1}(\vec{x})\right)^{\gamma}-N\left(B_{r}(\vec{x})\right)^{\gamma} \leq N\left(B_{4 \sqrt{d}+1}(\vec{x})\right)^{\gamma} \leq c_{d}^{\gamma}(5 \sqrt{d})^{d} .
$$

This together with (22) yields (21).

Fix $r>0$ and $\vec{x} \in \mathbb{R}^{d}$, if there exists $\vec{n} \in \mathbb{Z}^{d}$ such that $\vec{n} \in R_{r}(\vec{x})$. It follows easily from (16) and (19) that

$$
N\left(R_{r+1}(\vec{x})\right)^{\gamma}-N\left(R_{r}(\vec{x})\right)^{\gamma} \lesssim_{\gamma, d} G(r)^{\gamma-1 / d} .
$$

The following lemma is two refined summability estimates, which play key roles in our proofs.

Lemma 2.1 Let $R>\sqrt{d}$ and $\gamma>d$. Then

$$
\max \left\{\sum_{\substack{|\vec{n}| \geq R \\ \vec{n} \in \mathbb{Z}^{d}}}|\vec{n}|^{-\gamma}, \sum_{\substack{\|\vec{n}\|_{\infty} \geq R \\ \vec{n} \in \mathbb{Z}^{d}}}\|\vec{n}\|_{\infty}^{-\gamma}\right\} \lesssim d, \gamma R^{d-\gamma}
$$

Proof For any $\vec{n} \in \mathbb{Z}^{d}$, let $Q(\vec{n})=\left\{x \in \mathbb{R}^{d}:-1 / 2<x_{i}-n_{i} \leq 1 / 2,1 \leq i \leq d\right\}$. Clearly, $Q(\vec{n}) \cap$ $Q(\vec{l})=\emptyset$ for $\vec{n} \neq \vec{l}$ and $\bigcup_{|\vec{n}| \geq R, \vec{n} \in \mathbb{Z}^{d}} Q(\vec{n}) \subset\left\{x \in \mathbb{R}^{d}:|x| \geq R / 2\right\}$. When $\vec{x} \in Q(\vec{n})$ and $|\vec{n}| \geq R$, we have

$$
|\vec{x}| \leq|\vec{x}-\vec{n}|+|\vec{n}| \leq \sqrt{d} / 2+|\vec{n}| \leq 2|\vec{n}|
$$

It follows that

$$
\sum_{\substack{|\vec{n}| \geq R \\ \vec{n} \in \mathbb{Z}^{d}}}|\vec{n}|^{-\gamma} \leq \sum_{\substack{|\vec{n}| \geq R \\ \vec{n} \in \mathbb{Z}^{d}}} \int_{Q(\vec{n})}\left|\frac{x}{2}\right|^{-\gamma} d x \leq \int_{|x| \geq R / 2}\left|\frac{x}{2}\right|^{-\gamma} d x \lesssim d, \gamma R^{d-\gamma}
$$

Note that $\frac{|\vec{n}|}{\sqrt{d}} \leq\|\vec{n}\|_{\infty} \leq|\vec{n}|$. Then (24) leads to

$$
\sum_{\substack{\|\vec{n}\|_{\infty} \geq R \\ \vec{n} \in \mathbb{Z}^{d}}}\|\vec{n}\|_{\infty}^{-\gamma} \leq \sqrt{d}^{\gamma} \sum_{\substack{|\vec{n}| \geq R \\ \vec{n} \in \mathbb{Z}^{d}}}|\vec{n}|^{-\gamma} \lesssim d, \gamma R^{d-\gamma}
$$




\section{Proof of Theorem 1.5}

\subsection{Proof of Theorem 1.5 for $M_{\alpha, \mathcal{B}}^{\lambda}$}

The proof will be divided into two parts.

Step 1. Proof of the boundedness part. Let $0 \leq \beta \leq 1$ and $q \geq 1$ such that $q>\frac{d}{m d-\alpha+\beta}$. Let $\vec{f}=\left(f_{1}, \ldots, f_{m}\right)$ with each $f_{j} \in \ell^{1}\left(\mathbb{Z}^{d}\right)$. Without loss of generality we may assume that all $f_{j} \geq 0$. We want to show that

$$
\begin{aligned}
& \left\|D_{l} \mathrm{M}_{\alpha, \mathcal{B}}^{\lambda}(\vec{f})\right\|_{\ell q\left(\mathbb{Z}^{d}\right)}^{q} \\
& \quad \lesssim_{\alpha, m, d, q, \beta} \sum_{\mu=1}^{m}\left(\prod_{1 \leq j \neq \mu \leq m}\left\|f_{j}\right\|_{\ell^{1}\left(\mathbb{Z}^{d}\right)}^{q}\right)\left(\left\|D_{l} f_{\mu}\right\|_{\ell^{1}\left(\mathbb{Z}^{d}\right)}^{q(1-\beta)}\left\|f_{\mu}\right\|_{\ell^{1}\left(\mathbb{Z}^{d}\right)}^{q \beta}\right)
\end{aligned}
$$

for all $1 \leq l \leq d$. We shall prove (25) for $l=d$, and other cases are analogous. In what follows, we set $\vec{n}=\left(n^{\prime}, n_{d}\right) \in \mathbb{Z}^{d}$ with $n^{\prime}=\left(n_{1}, \ldots, n_{d-1}\right) \in \mathbb{Z}^{d-1}$. Then

$$
\left\|D_{d} \mathrm{M}_{\alpha, \mathcal{B}}^{\lambda}(\vec{f})\right\|_{\ell^{q}\left(\mathbb{Z}^{d}\right)}^{q}=\sum_{n^{\prime} \in \mathbb{Z}^{d-1}} \sum_{n_{d} \in \mathbb{Z}}\left|\mathrm{M}_{\alpha, \mathcal{B}}^{\lambda}(\vec{f})\left(n^{\prime}, n_{d}+1\right)-\mathrm{M}_{\alpha, \mathcal{B}}^{\lambda}(\vec{f})\left(n^{\prime}, n_{d}\right)\right|^{q} .
$$

For each $n^{\prime} \in \mathbb{Z}^{d-1}$, let

$$
\begin{aligned}
& X_{n^{\prime}}^{+}=\left\{n_{d} \in \mathbb{Z}: \mathrm{M}_{\alpha, \mathcal{B}}^{\lambda}(\vec{f})\left(n^{\prime}, n_{d}+1\right)<\mathrm{M}_{\alpha, \mathcal{B}}^{\lambda}(\vec{f})\left(n^{\prime}, n_{d}\right)\right\}, \\
& X_{n^{\prime}}^{-}=\left\{n_{d} \in \mathbb{Z}: \mathrm{M}_{\alpha, \mathcal{B}}^{\lambda}(\vec{f})\left(n^{\prime}, n_{d}+1\right)>\mathrm{M}_{\alpha, \mathcal{B}}^{\lambda}(\vec{f})\left(n^{\prime}, n_{d}\right)\right\}, \\
& X_{n^{\prime}}=\left\{n_{d} \in \mathbb{Z}: \mathrm{M}_{\alpha, \mathcal{B}}^{\lambda}(\vec{f})\left(n^{\prime}, n_{d}+1\right)=\mathrm{M}_{\alpha, \mathcal{B}}^{\lambda}(\vec{f})\left(n^{\prime}, n_{d}\right)\right\} .
\end{aligned}
$$

Hence,

$$
\begin{aligned}
& \left\|D_{d} \mathrm{M}_{\alpha, \mathcal{B}}^{\lambda}(\vec{f})\right\|_{\ell q\left(\mathbb{Z}^{d}\right)}^{q} \\
& =\sum_{n^{\prime} \in \mathbb{Z}^{d-1}} \sum_{n_{d} \in X_{n^{\prime}}^{+}}\left(\mathrm{M}_{\alpha, \mathcal{B}}^{\lambda}(\vec{f})\left(n^{\prime}, n_{d}\right)-\mathrm{M}_{\alpha, \mathcal{B}}^{\lambda}(\vec{f})\left(n^{\prime}, n_{d}+1\right)\right)^{q} \\
& \quad+\sum_{n^{\prime} \in \mathbb{Z}^{d-1}} \sum_{n_{d} \in X_{n^{\prime}}^{-}}\left(\mathrm{M}_{\alpha, \mathcal{B}}^{\lambda}(\vec{f})\left(n^{\prime}, n_{d}+1\right)-\mathrm{M}_{\alpha, \mathcal{B}}^{\lambda}(\vec{f})\left(n^{\prime}, n_{d}\right)\right)^{q} .
\end{aligned}
$$

Thus, to prove (25), it suffices to prove that

$$
\begin{aligned}
& \sum_{n^{\prime} \in \mathbb{Z}^{d-1}} \sum_{n_{d} \in X_{n^{\prime}}^{+}}\left(\mathrm{M}_{\alpha, \mathcal{B}}^{\lambda}(\vec{f})\left(n^{\prime}, n_{d}\right)-\mathrm{M}_{\alpha, \mathcal{B}}^{\lambda}(\vec{f})\left(n^{\prime}, n_{d}+1\right)\right)^{q} \\
& \lesssim_{\alpha, m, d, q, \beta, \lambda} \sum_{\mu=1}^{m}\left(\prod_{1 \leq j \neq \mu \leq m}\left\|f_{j}\right\|_{\ell^{1}\left(\mathbb{Z}^{d}\right)}^{q}\right)\left(\left\|D_{d} f_{\mu}\right\|_{\ell^{1}\left(\mathbb{Z}^{d}\right)}^{q(1-\beta)}\left\|f_{\mu}\right\|_{\ell^{1}\left(\mathbb{Z}^{d}\right)}^{q \beta}\right) ; \\
& \sum_{n^{\prime} \in \mathbb{Z}^{d-1}} \sum_{n_{d} \in X_{n^{\prime}}^{-}}\left(\mathrm{M}_{\alpha, \mathcal{B}}^{\lambda}(\vec{f})\left(n^{\prime}, n_{d}+1\right)-\mathrm{M}_{\alpha, \mathcal{B}}^{\lambda}(\vec{f})\left(n^{\prime}, n_{d}\right)\right)^{q} \\
& \lesssim_{\alpha, m, d, q, \beta, \lambda} \sum_{\mu=1}^{m}\left(\prod_{1 \leq j \neq \mu \leq m}\left\|f_{j}\right\|_{\ell^{1}\left(\mathbb{Z}^{d}\right)}^{q}\right)\left(\left\|D_{d} f_{\mu}\right\|_{\ell^{1}\left(\mathbb{Z}^{d}\right)}^{q(1-\beta)}\left\|f_{\mu}\right\|_{\ell^{1}\left(\mathbb{Z}^{d}\right)}^{q \beta}\right) .
\end{aligned}
$$


We only prove (26) since (27) is analogous. For $r>0$, we define the function $\mathrm{A}_{r}(\vec{f}): \mathbb{R}^{d} \rightarrow$ $\mathbb{R}$ by

$$
\mathrm{A}_{r}(\vec{f})(\vec{x})=\frac{1}{N\left(B_{r}(\vec{x})\right)^{m-\frac{\alpha}{d}}} \prod_{j=1}^{m} \sum_{\vec{k} \in B_{r}(\vec{x}) \cap \mathbb{Z}^{d}} f_{j}(\vec{k}) \quad \forall \vec{x} \in \mathbb{R}^{d}
$$

Since all $f_{j} \in \ell^{1}\left(\mathbb{Z}^{d}\right)$, then, for any $\vec{x} \in \mathbb{R}^{d}, \lim _{r \rightarrow \infty} \mathrm{A}_{r}(\vec{f})(\vec{x})=0$. It follows that, for any $\left(n^{\prime}, n_{d}\right) \in \mathbb{Z}^{d}$ with $X_{n^{\prime}}^{+}$, there exist $\vec{x} \in \mathbb{R}^{d}$ and $r\left(n^{\prime}, n_{d}\right)>0$ such that $\left|\left(n^{\prime}, n_{d}\right)-\vec{x}\right| \leq \lambda r\left(n^{\prime}, n_{d}\right)$ and $\mathrm{M}_{\alpha, \mathcal{B}}^{\lambda}(\vec{f})\left(n^{\prime}, n_{d}\right)=\mathrm{A}_{r\left(n^{\prime}, n_{d}\right)}(\vec{f})(\vec{x})$. Note that $\left|\left(n^{\prime}, n_{d}+1\right)-\left(\vec{x}+\vec{e}_{d}\right)\right| \leq \lambda r\left(n^{\prime}, n_{d}\right)$. Consequently,

$$
\begin{aligned}
& \mathrm{M}_{\alpha, \mathcal{B}}^{\lambda}(\vec{f})\left(n^{\prime}, n_{d}+1\right) \\
& \quad \geq \frac{1}{N\left(B_{r\left(n^{\prime}, n_{d}\right)+1}\left(\vec{x}+\vec{e}_{d}\right)\right)^{m-\frac{\alpha}{d}}} \prod_{j=1}^{m} \sum_{\vec{k} \in B_{r\left(n^{\prime}, n_{d}\right)+1}\left(\vec{x}+\vec{e}_{d}\right) \cap \mathbb{Z}^{d}} f_{j}(\vec{k}) \\
& \quad \geq \frac{1}{N\left(B_{r\left(n^{\prime}, n_{d}\right)+1}\left(\vec{x}+\vec{e}_{d}\right)\right)^{m-\frac{\alpha}{d}}} \prod_{j=1}^{m} \sum_{\vec{k} \in B_{r\left(n^{\prime}, n_{d}\right)}(\vec{x}) \cap \mathbb{Z}^{d}} f_{j}(\vec{k}) \\
& \quad \geq \frac{N\left(B_{r\left(n^{\prime}, n_{d}\right)}(\vec{x})\right)^{m-\frac{\alpha}{d}}}{N\left(B_{r\left(n^{\prime}, n_{d}\right)+1}(\vec{x})\right)^{m-\frac{\alpha}{d}}} \mathrm{~A}_{r\left(n^{\prime}, n_{d}\right)}(\vec{f})(\vec{x}) \\
& \quad \geq \frac{N\left(B_{r\left(n^{\prime}, n_{d}\right)}(\vec{x})\right)^{m}}{N\left(B_{r\left(n^{\prime}, n_{d}\right)+1}(\vec{x})\right)^{m}} \mathrm{M}_{\alpha, \mathcal{B}}^{\lambda}(\vec{f})\left(n^{\prime}, n_{d}\right) .
\end{aligned}
$$

This together with (20) and (21) implies that

$$
\begin{aligned}
& \mathrm{M}_{\alpha, \mathcal{B}}^{\lambda}(\vec{f})\left(n^{\prime}, n_{d}\right)-\mathrm{M}_{\alpha, \mathcal{B}}^{\lambda}(\vec{f})\left(n^{\prime}, n_{d}+1\right) \\
& \quad \leq\left(1-\frac{N\left(B_{r\left(n^{\prime}, n_{d}\right)}(\vec{x})\right)^{m}}{N\left(B_{r\left(n^{\prime}, n_{d}\right)+1}(\vec{x})\right)^{m}}\right) \frac{1}{N\left(B_{r\left(n^{\prime}, n_{d}\right)}(\vec{x})\right)^{m-\frac{\alpha}{d}}} \prod_{j=1}^{m} \sum_{\vec{k} \in B_{r\left(n^{\prime}, n_{d}\right)}(\vec{x}) \cap \mathbb{Z}^{d}} f_{j}(\vec{k}) \\
& \quad{ }_{m, d} F\left(r\left(n^{\prime}, n_{d}\right)\right)^{\frac{\alpha-1}{d}-m} \prod_{j=1}^{m} \sum_{\vec{k} \in B_{r\left(n^{\prime}, n_{d}\right)}(\vec{x}) \cap \mathbb{Z}^{d}} f_{j}(\vec{k}) .
\end{aligned}
$$

On the other hand, (20) yields that

$$
\begin{aligned}
& \mathrm{M}_{\alpha, \mathcal{B}}^{\lambda}(\vec{f})\left(n^{\prime}, n_{d}\right)-\mathrm{M}_{\alpha, \mathcal{B}}^{\lambda}(\vec{f})\left(n^{\prime}, n_{d}+1\right) \\
& \leq \mathrm{A}_{r\left(n^{\prime}, n_{d}\right)}(\vec{f})(\vec{x})-\mathrm{A}_{r\left(n^{\prime}, n_{d}\right)}(\vec{f})\left(\vec{x}+\vec{e}_{d}\right) \\
& =\frac{1}{N\left(B_{r\left(n^{\prime}, n_{d}\right)}(\vec{x})\right)^{m-\frac{\alpha}{d}}} \prod_{j=1}^{m} \sum_{\vec{k} \in B_{r\left(n^{\prime}, n_{d}\right)}(\vec{x}) \cap \mathbb{Z}^{d}} f_{j}(\vec{k}) \\
& \quad-\frac{1}{N\left(B_{r\left(n^{\prime}, n_{d}\right)}\left(\vec{x}+\vec{e}_{d}\right)\right)^{m-\frac{\alpha}{d}}} \prod_{j=1}^{m} \sum_{\vec{k} \in B_{r\left(n^{\prime}, n_{d}\right)}\left(\vec{x}+\vec{e}_{d}\right) \cap \mathbb{Z}^{d}} f_{j}(\vec{k}) \\
& \leq F\left(r\left(n^{\prime}, n_{d}\right)\right)^{\frac{\alpha}{d}-m} \sum_{\mu=1}^{m} \sum_{\vec{k} \in B_{r\left(n^{\prime}, n_{d}\right)}(\vec{x}) \cap \mathbb{Z}^{d}}\left|D_{d} f_{\mu}(\vec{k})\right| \prod_{1 \leq j \neq \mu \leq m}\left\|f_{j}\right\|_{\ell^{1}\left(\mathbb{Z}^{d}\right)} .
\end{aligned}
$$


Note that $B_{\vec{r}\left(n^{\prime}, n_{d}\right)}(\vec{x}) \subset B_{(\lambda+1) \vec{r}\left(n^{\prime}, n_{d}\right)}\left(n^{\prime}, n_{d}\right)$. It follows from (28) and (29) that

$$
\begin{aligned}
& \left(\mathrm{M}_{\alpha, \mathcal{B}}^{\lambda}(\vec{f})\left(n^{\prime}, n_{d}\right)-\mathrm{M}_{\alpha, \mathcal{B}}^{\lambda}(\vec{f})\left(n^{\prime}, n_{d}+1\right)\right)^{q} \\
& \quad \sum_{m, d}\left(F\left(r\left(n^{\prime}, n_{d}\right)\right)^{\frac{\alpha}{d}-m} \sum_{\mu=1}^{m} \sum_{\vec{k} \in B_{(\lambda+1) r\left(n^{\prime}, n_{d}\right)}\left(n^{\prime}, n_{d}\right) \cap \mathbb{Z}^{d}}\left|D_{d} f_{\mu}(\vec{k})\right|\right. \\
& \left.\quad \times \prod_{1 \leq j \neq \mu \leq m}\left\|f_{j}\right\|_{\ell^{1}\left(\mathbb{Z}^{d}\right)}\right)^{q(1-\beta)} \\
& \quad \times\left(F\left(r\left(n^{\prime}, n_{d}\right)\right)^{\frac{\alpha-1}{d}-m} \prod_{j=1}^{m} \sum_{\vec{k} \in B_{(\lambda+1) r\left(n^{\prime}, n_{d}\right)}\left(n^{\prime}, n_{d}\right) \cap \mathbb{Z}^{d}} f_{j}(\vec{k})\right)^{q \beta} .
\end{aligned}
$$

For convenience, fix $1 \leq \mu \leq m$, we set

$$
A_{\mu}:=\left(\prod_{1 \leq j \neq \mu \leq m}\left\|f_{j}\right\|_{\ell^{1}\left(\mathbb{Z}^{d}\right)}^{q}\right)\left(\left\|D_{d} f_{\mu}\right\|_{\ell^{1}\left(\mathbb{Z}^{d}\right)}^{(q-1)(1-\beta)}\left\|f_{\mu}\right\|_{\ell^{1}\left(\mathbb{Z}^{d}\right)}^{(q-1) \beta}\right) .
$$

Then (30) leads to

$$
\begin{aligned}
& \left(\mathrm{M}_{\alpha, \mathcal{B}}^{\lambda}(\vec{f})\left(n^{\prime}, n_{d}\right)-\mathrm{M}_{\alpha, \mathcal{B}}^{\lambda}(\vec{f})\left(n^{\prime}, n_{d}+1\right)\right)^{q} \\
& \lesssim_{m, d} \sum_{\mu=1}^{m} A_{\mu}\left(F\left(r\left(n^{\prime}, n_{d}\right)\right)\right)^{-\frac{q(m d-\alpha+\beta)}{d}} \\
& \quad \times\left(\sum_{\vec{k} \in B_{(\lambda+1) r\left(n^{\prime}, n_{d}\right)}\left(n^{\prime}, n_{d}\right) \cap \mathbb{Z}^{d}}\left|D_{d} f_{\mu}(\vec{k})\right|\right)^{(1-\beta)} \\
& \left.\quad \times \sum_{\vec{k} \in B_{(\lambda+1) r\left(n^{\prime}, n_{d}\right)}\left(n^{\prime}, n_{d}\right) \cap \mathbb{Z}^{d}} f_{\mu}(\vec{k})\right)^{\beta} .
\end{aligned}
$$

By (31) and Hölder's inequality with exponents $p=\frac{1}{1-\beta}$ and $p^{\prime}=\frac{1}{\beta}$,

$$
\begin{aligned}
& \sum_{n^{\prime} \in \mathbb{Z}^{d-1}} \sum_{n_{d} \in X_{n^{\prime}}^{+}}\left(\mathrm{M}_{\alpha, \mathcal{B}}^{\lambda}(\vec{f})\left(n^{\prime}, n_{d}\right)-\mathrm{M}_{\alpha, \mathcal{B}}^{\lambda}(\vec{f})\left(n^{\prime}, n_{d}+1\right)\right)^{q} \\
& \lesssim_{m, d} \sum_{\mu=1}^{m} A_{\mu}\left(\sum_{n^{\prime} \in \mathbb{Z}^{d-1}} \sum_{n_{d} \in X_{n^{\prime}}^{+}}\left(F\left(r\left(n^{\prime}, n_{d}\right)\right)\right)^{-\frac{q(m d-\alpha+\beta)}{d}}\right. \\
& \left.\quad \times \sum_{\vec{k} \in B_{(\lambda+1) r\left(n^{\prime}, n_{d}\right)}\left(n^{\prime}, n_{d}\right) \cap \mathbb{Z}^{d}}\left|D_{d} f_{\mu}(\vec{k})\right|\right)^{1-\beta} \\
& \quad \times\left(\sum_{n^{\prime} \in \mathbb{Z}^{d-1}} \sum_{n_{d} \in X_{n^{\prime}}^{+}}\left(F\left(r\left(n^{\prime}, n_{d}\right)\right)\right)^{-\frac{q(m d-\alpha+\beta)}{d}} \sum_{\vec{k} \in B_{(\lambda+1) r\left(n^{\prime}, n_{d}\right)}\left(n^{\prime}, n_{d}\right) \cap \mathbb{Z}^{d}} f_{\mu}(\vec{k})\right)^{\beta} .
\end{aligned}
$$


Note that

$$
\begin{aligned}
& \sum_{n^{\prime} \in \mathbb{Z}^{d-1}} \sum_{n_{d} \in X_{n^{\prime}}^{+}}\left(F\left(r\left(n^{\prime}, n_{d}\right)\right)\right)^{-\frac{q(m d-\alpha+\beta)}{d}} \sum_{\vec{k} \in B_{(\lambda+1) r\left(n^{\prime}, n_{d}\right)}\left(n^{\prime}, n_{d}\right) \cap \mathbb{Z}^{d}}\left|D_{d} f_{\mu}(\vec{k})\right| \\
& \leq \sum_{\vec{k} \in \mathbb{Z}^{d}}\left|D_{d} f_{\mu}(\vec{k})\right| \sum_{\vec{n} \in \mathbb{Z}^{d}}(F(r(\vec{n})))^{-\frac{q(m d-\alpha+\beta)}{d}} \chi_{\{|\vec{k}-\vec{n}| \leq(\lambda+1) r(\vec{n})\}} .
\end{aligned}
$$

Fix $\vec{k} \in \mathbb{Z}^{d}$. By (15) we have

$$
\sum_{\vec{n} \in \mathbb{Z}^{d}} \chi_{\{|\vec{k}-\vec{n}|<4 \sqrt{d}(\lambda+1)\}} \leq c_{d}(4 \sqrt{d}(\lambda+1)+\sqrt{d} / 2)^{d} .
$$

Since $q(m d-\alpha+\beta)>d$. Invoking Lemma 2.1, we have

$$
\begin{aligned}
& \sum_{\vec{n} \in \mathbb{Z}^{d}}\left(\frac{|\vec{k}-\vec{n}|}{\lambda+1}-\sqrt{d}\right)^{-q(m d-\alpha+\beta)} \chi_{\{|\vec{k}-\vec{n}| \geq 4 \sqrt{d}(\lambda+1)\}} \\
& \lesssim_{\alpha, m, d, \beta, q, \lambda} \sum_{\substack{|\vec{n}| \geq 4 \sqrt{d}(\lambda+1) \\
\vec{n} \in \mathbb{Z}^{d}}}|\vec{n}|^{-q(m d-\alpha+\beta)} \lesssim_{\alpha, m, d, \beta, q, \lambda}(4 \sqrt{d}(\lambda+1))^{d-q(m d-\alpha+\beta)} .
\end{aligned}
$$

Combining (35) with (34) yields that

$$
\begin{aligned}
& \sum_{\vec{n} \in \mathbb{Z}^{d}}(F(r(\vec{n})))^{-\frac{q(m d-\alpha+\beta)}{d}} \chi_{\{|\vec{k}-\vec{n}| \leq(\lambda+1) r(\vec{n})\}} \\
& \leq \sum_{\vec{n} \in \mathbb{Z}^{d}}(F(r(\vec{n})))^{-\frac{q(m d-\alpha+\beta)}{d}} \chi_{\{|\vec{k}-\vec{n}|<4 \sqrt{d}(\lambda+1)\}} \\
& \quad+\sum_{\vec{n} \in \mathbb{Z}^{d}}(F(r(\vec{n})))^{-\frac{q(m d-\alpha+\beta)}{d}} \chi_{\{4 \sqrt{d}(\lambda+1) \leq|\vec{k}-\vec{n}| \leq(\lambda+1) r(\vec{n})\}} \\
& \leq \sum_{\vec{n} \in \mathbb{Z}^{d}} \chi_{\{|\vec{k}-\vec{n}|<4 \sqrt{d}(\lambda+1)\}} \\
& \quad+\sum_{\vec{n} \in \mathbb{Z}^{d}} c_{d}\left(\frac{|\vec{k}-\vec{n}|}{\lambda+1}-\sqrt{d}\right)^{-q(m d-\alpha+\beta)} \chi_{\{|\vec{k}-\vec{n}| \geq 4 \sqrt{d}(\lambda+1)\}} \\
& \lesssim_{\alpha, m, d, \beta, q, \lambda} 1 .
\end{aligned}
$$

(36) together with (33) yields that

$$
\begin{aligned}
& \sum_{n^{\prime} \in \mathbb{Z}^{d-1}} \sum_{n_{d} \in X_{n^{\prime}}^{+}}\left(F\left(r\left(n^{\prime}, n_{d}\right)\right)^{-\frac{q(m d-\alpha+\beta)}{d}} \sum_{\vec{k} \in B_{(\lambda+1) r\left(n^{\prime}, n_{d}\right)}\left(n^{\prime}, n_{d}\right) \cap \mathbb{Z}^{d}}\left|D_{d} f_{\mu}(\vec{k})\right|\right. \\
& \lesssim_{\alpha, m, d, \beta, q, \lambda}\left\|D_{d} f_{\mu}\right\|_{\ell^{1}\left(\mathbb{R}^{d}\right)^{.}}
\end{aligned}
$$

Similarly,

$$
\sum_{n^{\prime} \in \mathbb{Z}^{d-1}} \sum_{n_{d} \in X_{n^{\prime}}^{+}}\left(F\left(r\left(n^{\prime}, n_{d}\right)\right)^{-\frac{q(m d-\alpha+\beta)}{d}} \sum_{\vec{k} \in B_{(\lambda+1) r\left(n^{\prime}, n_{d}\right)}\left(n^{\prime}, n_{d}\right) \cap \mathbb{Z}^{d}}\left|f_{\mu}(\vec{k})\right|\right.
$$




$$
\lesssim_{\alpha, m, d, \beta, q, \lambda}\left\|f_{\mu}\right\|_{\ell^{1}\left(\mathbb{R}^{d}\right)}
$$

Then (26) follows from (32) and (37)-(38).

Step 2. Proof of the continuity part. Let $\vec{f}=\left(f_{1}, \ldots, f_{m}\right)$ with each $f_{j} \in \ell^{1}\left(\mathbb{Z}^{d}\right)$ and $g_{i, j} \rightarrow f_{j}$ in $\ell^{1}\left(\mathbb{Z}^{d}\right)$ for any $1 \leq j \leq m$ as $i \rightarrow \infty$. Let $\vec{g}_{i}=\left(g_{i, 1}, \ldots, g_{i, m}\right)$ for $i \in \mathbb{Z}$. Since ||$g_{i, j}|-| f_{j}|| \leq\left|g_{i, j}-f_{j}\right|$ for all $1 \leq j \leq d$, we may assume without loss of generality that all $g_{i, j} \geq 0$ and $f_{j} \geq 0$. It suffices to show that

$$
\lim _{i \rightarrow \infty}\left\|D_{l} \mathrm{M}_{\alpha, \mathcal{B}}^{\lambda}\left(\vec{g}_{i}\right)-D_{l} \mathrm{M}_{\alpha, \mathcal{B}}^{\lambda}(\vec{f})\right\|_{\ell q}\left(\mathbb{Z}^{d}\right)=0
$$

for each $l=1,2, \ldots, d$.

We only prove (39) for $l=d$ (since other cases are analogous). By the boundedness part, we see that $D_{d} \mathrm{M}_{\alpha, \mathcal{B}}^{\lambda}(\vec{f}) \in \ell^{q}\left(\mathbb{Z}^{d}\right)$. Then, fix $\epsilon \in(0,1)$, there exist $N_{1}=N_{1}(\epsilon, \vec{f})>0$ and $\Lambda>$ $8 \sqrt{d}(\lambda+1)$ such that

$$
\begin{aligned}
& \left\|g_{i, j}-f_{j}\right\|_{\ell^{1}\left(\mathbb{Z}^{d}\right)}<\epsilon \text { and }\left\|g_{i, j}\right\|_{\ell^{1}\left(\mathbb{Z}^{d}\right)} \leq\left\|f_{j}\right\|_{\ell^{1}\left(\mathbb{Z}^{d}\right)}+1 \quad \forall i \geq N_{1} \text { and } 1 \leq j \leq m ; \\
& \max \left\{\left\|D_{d} \mathrm{M}_{\alpha, \mathcal{B}}^{\lambda}(\vec{f}) \chi_{\left(B_{3 \Lambda}(\overrightarrow{0})\right)^{c}}\right\|_{\ell^{q}\left(\mathbb{Z}^{d}\right)^{\prime}} \sup _{1 \leq j \leq m}\left\|f_{j} \chi_{\left(B_{\Lambda}(\overrightarrow{0})\right)^{c}}\right\|_{\ell^{1}\left(\mathbb{Z}^{d}\right)}, \Lambda^{d-q(m d-\alpha+\beta)}\right\}<\epsilon .
\end{aligned}
$$

Combining (41) with (40) yields that

$$
\begin{aligned}
& \sup _{1 \leq j \leq m}\left\|D_{d} g_{i, j} \chi_{\left(B_{\Lambda}(\overrightarrow{0})\right) c}\right\|_{\ell^{1}\left(\mathbb{Z}^{d}\right)} \\
& \quad \leq 2 \sup _{1 \leq j \leq m}\left\|g_{i, j} \chi_{\left(B_{\Lambda}(\overrightarrow{0})\right)^{c}}\right\|_{\ell^{1}\left(\mathbb{Z}^{d}\right)} \\
& \quad \leq 2 \sup _{1 \leq j \leq m}\left(\left\|g_{i, j}-f_{j}\right\|_{\ell^{1}\left(\mathbb{Z}^{d}\right)}+\left\|f_{j} \chi_{\left(B_{\Lambda}(\overrightarrow{0})\right)}\right\|_{\ell^{1}\left(\mathbb{Z}^{d}\right)}\right) \leq 4 \epsilon \quad \forall i \geq N_{1} .
\end{aligned}
$$

For any $\vec{n} \in \mathbb{Z}^{d}$ and $i \geq N_{1}$, we can write

$$
\begin{aligned}
& \left|\mathrm{M}_{\alpha, \mathcal{B}}^{\lambda}\left(\vec{g}_{i}\right)(\vec{n})-\mathrm{M}_{\alpha, \mathcal{B}}^{\lambda}(\vec{f})(\vec{n})\right| \\
& \quad \leq \sup _{\substack{r>0, \vec{x} \in \mathbb{R}^{d} \\
|\vec{n}-\vec{x}| \leq \lambda r}} \frac{1}{N\left(B_{r}(\vec{x})\right)^{m-\frac{\alpha}{d}}}\left|\prod_{j=1}^{m} \sum_{\vec{k} \in B_{r}(\vec{x}) \cap \mathbb{Z}^{d}} g_{i, j}(\vec{k})-\prod_{j=1}^{m} \sum_{\vec{k} \in B_{r}(\vec{x}) \cap \mathbb{Z}^{d}} f_{j}(\vec{k})\right| \\
& \quad \leq \sum_{l=1}^{m}\left(\prod_{j=1}^{l-1}\left\|f_{j}\right\|_{\ell^{1}\left(\mathbb{Z}^{d}\right)}\right)\left(\prod_{\mu=l+1}^{m}\left\|g_{i, \mu}\right\|_{\ell^{1}\left(\mathbb{Z}^{d}\right)}\right)\left\|g_{i, l}-f_{l}\right\|_{\ell^{1}\left(\mathbb{Z}^{d}\right)},
\end{aligned}
$$

which together with (40) implies that $\mathrm{M}_{\alpha, \mathcal{B}}^{\lambda}\left(\vec{g}_{i}\right)(\vec{n}) \rightarrow \mathrm{M}_{\alpha, \mathcal{B}}^{\lambda}(\vec{f})(\vec{n})$ as $i \rightarrow \infty$ for any $\vec{n} \in \mathbb{Z}^{d}$. Consequently,

$$
D_{d} \mathrm{M}_{\alpha, \mathcal{B}}^{\lambda}\left(\vec{g}_{i}\right)(\vec{n}) \rightarrow D_{d} \mathrm{M}_{\alpha, \mathcal{B}}^{\lambda}(\vec{f})(\vec{n}) \quad \text { as } i \rightarrow \infty \forall \vec{n} \in \mathbb{Z}^{d}
$$

By (43), there exists $N_{2}=N_{2}(\epsilon, \Lambda)>0$ such that

$$
\left|D_{d} \mathrm{M}_{\alpha, \mathcal{B}}^{\lambda}\left(\vec{g}_{i}\right)(\vec{n})-D_{d} \mathrm{M}_{\alpha, \mathcal{B}}^{\lambda}(\vec{f})(\vec{n})\right| \leq \frac{\epsilon}{\left(N\left(B_{3 \Lambda}(\overrightarrow{0})\right)\right)^{1 / q}} \quad \forall i \geq N_{2} \text { and }|\vec{n}|<3 \Lambda
$$


It follows from (44) that

$$
\begin{aligned}
\left\|D_{l} \mathrm{M}_{\alpha, \mathcal{B}}^{\lambda}\left(\vec{g}_{i}\right)-D_{l} \mathrm{M}_{\alpha, \mathcal{B}}^{\lambda}(\vec{f})\right\|_{\ell q\left(\mathbb{Z}^{d}\right)} \\
\leq\left\|\left(D_{l} \mathrm{M}_{\alpha, \mathcal{B}}^{\lambda}\left(\vec{g}_{i}\right)-D_{l} \mathrm{M}_{\alpha, \mathcal{B}}^{\lambda}(\vec{f})\right) \chi_{B_{3 \Lambda}(\overrightarrow{0})}\right\|_{\ell q\left(\mathbb{Z}^{d}\right)} \\
\quad+\left\|\left(D_{l} \mathrm{M}_{\alpha, \mathcal{B}}^{\lambda}\left(\vec{g}_{i}\right)-D_{l} \mathrm{M}_{\alpha, \mathcal{B}}^{\lambda}(\vec{f})\right) \chi_{\left(B_{3 \Lambda}(\overrightarrow{0})\right)^{c}}\right\|_{\ell q\left(\mathbb{Z}^{d}\right)} \\
\leq \epsilon+\left\|\left(D_{l} \mathrm{M}_{\alpha, \mathcal{B}}^{\lambda}\left(\vec{g}_{i}\right)-D_{l} \mathrm{M}_{\alpha, \mathcal{B}}^{\lambda}(\vec{f})\right) \chi_{\left(B_{3 \Lambda}(\overrightarrow{0})\right)^{c}}\right\|_{\ell q\left(\mathbb{Z}^{d}\right)} \quad \forall i \geq N_{2} .
\end{aligned}
$$

Fix $i \geq \max \left\{N_{1}, N_{2}\right\}$. We can write

$$
\begin{aligned}
& \left\|\left(D_{l} \mathrm{M}_{\alpha, \mathcal{B}}^{\lambda}\left(\vec{g}_{i}\right)-D_{l} \mathrm{M}_{\alpha, \mathcal{B}}^{\lambda}(\vec{f})\right) \chi_{\left(B_{3 \Lambda}(\overrightarrow{0})\right)^{c}}\right\|_{\ell q\left(\mathbb{Z}^{d}\right)} \\
& \leq \sum_{\substack{\left|n^{\prime}\right| \geq 2 \Lambda \\
n^{\prime} \in \mathbb{Z}^{d-1}}} \sum_{n_{d} \in \mathbb{Z}}\left|D_{d} \mathrm{M}_{\alpha, \mathcal{B}}^{\lambda}\left(\vec{g}_{i}\right)(\vec{n})\right|^{q}+\sum_{\substack{n^{\prime} \in \mathbb{Z}^{d} \\
\left|n_{d}\right| \geq 2 \Lambda \\
n_{d} \in \mathbb{Z}}}\left|D_{d} \mathrm{M}_{\alpha, \mathcal{B}}^{\lambda}\left(\vec{g}_{i}\right)(\vec{n})\right|^{q} \\
& =: A_{1}+A_{2} \text {. }
\end{aligned}
$$

We first estimate $A_{1}$. For each $n^{\prime} \in \mathbb{Z}^{d-1}$ with $\left|n^{\prime}\right| \geq 2 \Lambda$, let

$$
\begin{aligned}
& Y_{n^{\prime}}^{+}=\left\{n_{d} \in \mathbb{Z}: \mathrm{M}_{\alpha, \mathcal{B}}^{\lambda}\left(\vec{g}_{i}\right)\left(n^{\prime}, n_{d}+1\right)<\mathrm{M}_{\alpha, \mathcal{B}}^{\lambda}\left(\vec{g}_{i}\right)\left(n^{\prime}, n_{d}\right)\right\}, \\
& Y_{n^{\prime}}^{-}=\left\{n_{d} \in \mathbb{Z}: \mathrm{M}_{\alpha, \mathcal{B}}^{\lambda}\left(\vec{g}_{i}\right)\left(n^{\prime}, n_{d}+1\right)>\mathrm{M}_{\alpha, \mathcal{B}}^{\lambda}\left(\vec{g}_{i}\right)\left(n^{\prime}, n_{d}\right)\right\}, \\
& Y_{n^{\prime}}=\left\{n_{d} \in \mathbb{Z}: \mathrm{M}_{\alpha, \mathcal{B}}^{\lambda}\left(\vec{g}_{i}\right)\left(n^{\prime}, n_{d}+1\right)=\mathrm{M}_{\alpha, \mathcal{B}}^{\lambda}\left(\vec{g}_{i}\right)\left(n^{\prime}, n_{d}\right)\right\} .
\end{aligned}
$$

Then

$$
\begin{aligned}
A_{1} \leq & \sum_{\substack{\left|n^{\prime}\right| \geq 2 \Lambda \\
n^{\prime} \in \mathbb{Z}^{d-1}}} \sum_{n_{d} \in Y_{n^{\prime}}^{+}}\left(\mathrm{M}_{\alpha, \mathcal{B}}^{\lambda}\left(\vec{g}_{i}\right)\left(n^{\prime}, n_{d}\right)-\mathrm{M}_{\alpha, \mathcal{B}}^{\lambda}\left(\vec{g}_{i}\right)\left(n^{\prime}, n_{d}+1\right)\right)^{q} \\
& +\sum_{\substack{\left|n^{\prime}\right| \geq 2 \Lambda \\
n^{\prime} \in \mathbb{Z}^{d-1}}} \sum_{n_{d} \in Y_{n^{\prime}}^{-}}\left(\mathrm{M}_{\alpha, \mathcal{B}}^{\lambda}\left(\vec{g}_{i}\right)\left(n^{\prime}, n_{d}+1\right)-\mathrm{M}_{\alpha, \mathcal{B}}^{\lambda}\left(\vec{g}_{i}\right)\left(n^{\prime}, n_{d}\right)\right)^{q} .
\end{aligned}
$$

We now prove that

$$
\begin{aligned}
& \sum_{\substack{\left|n^{\prime}\right| \geq 2 \Lambda \\
n \mathbb{Z}^{d-1}}} \sum_{n_{d} \in Y_{n^{\prime}}^{+}}\left(\mathrm{M}_{\alpha, \mathcal{B}}^{\lambda}\left(\vec{g}_{i}\right)\left(n^{\prime}, n_{d}\right)-\mathrm{M}_{\alpha, \mathcal{B}}^{\lambda}\left(\vec{g}_{i}\right)\left(n^{\prime}, n_{d}+1\right)\right)^{q} \lesssim_{\alpha, m, d, \beta, q, \lambda, \vec{f}} \epsilon ; \\
& \sum_{\substack{\left|n^{\prime}\right| \geq 2 \Lambda \\
n^{\prime} \in \mathbb{Z}^{d-1}}} \sum_{n_{d} \in Y_{n^{\prime}}^{-}}\left(\mathrm{M}_{\alpha, \mathcal{B}}^{\lambda}\left(\vec{g}_{i}\right)\left(n^{\prime}, n_{d}+1\right)-\mathrm{M}_{\alpha, \mathcal{B}}^{\lambda}\left(\vec{g}_{i}\right)\left(n^{\prime}, n_{d}\right)\right)^{q} \lesssim_{\alpha, m, d, \beta, q, \lambda, \vec{f}} \epsilon .
\end{aligned}
$$

We only prove (48), and (49) is analogous. Since all $g_{i, j} \in \ell^{1}\left(\mathbb{Z}^{d}\right)$, then for any $\left(n^{\prime}, n_{d}\right) \in \mathbb{Z}^{d}$ with $n_{d} \in Y_{n^{\prime}}^{+}$, there exist $\vec{x} \in \mathbb{R}^{d}$ and $r\left(n^{\prime}, n_{d}\right)>0$ such that $\left|\left(n^{\prime}, n_{d}\right)-\vec{x}\right| \leq \lambda r\left(n^{\prime}, n_{d}\right)$ and $\mathrm{M}_{\alpha, \mathcal{B}}^{\lambda}\left(\vec{g}_{i}\right)\left(n^{\prime}, n_{d}\right)=\mathrm{A}_{r\left(n^{\prime}, n_{d}\right)}\left(\vec{g}_{i}\right)(\vec{x})$. By the argument similar to those used in deriving (32),

$$
\sum_{\substack{\left|n^{\prime}\right| \geq 2 \Lambda \\ n^{\prime} \in \mathbb{Z}^{d-1}}} \sum_{n_{d} \in Y_{n^{\prime}}^{+}}\left(\mathrm{M}_{\alpha, \mathcal{B}}^{\lambda}\left(\vec{g}_{i}\right)\left(n^{\prime}, n_{d}\right)-\mathrm{M}_{\alpha, \mathcal{B}}^{\lambda}\left(\vec{g}_{i}\right)\left(n^{\prime}, n_{d}+1\right)\right)^{q}
$$




$$
\begin{aligned}
& \lesssim_{m, d} \sum_{\mu=1}^{m}\left(\prod_{1 \leq j \neq \mu \leq m}\left\|g_{i, j}\right\|_{\ell^{1}\left(\mathbb{Z}^{d}\right)}^{q}\right)\left(\left\|D_{d} g_{i, \mu}\right\|_{\ell^{1}\left(\mathbb{Z}^{d}\right)}^{(q-1)(1-\beta)}\left\|g_{i, \mu}\right\|_{\ell^{1}\left(\mathbb{Z}^{d}\right)}^{(q-1) \beta}\right) \\
& \times\left(\sum _ { \substack { | n ^ { \prime } | \geq 2 \Lambda \\
n ^ { \prime } \in \mathbb { Z } ^ { d - 1 } } } \sum _ { n _ { d } \in Y _ { n ^ { \prime } } ^ { + } } \left(F\left(r\left(n^{\prime}, n_{d}\right)\right)^{-\frac{q(m d-\alpha+\beta)}{d}}\right.\right. \\
& \left.\times \sum_{\vec{k} \in B_{(\lambda+1) r\left(n^{\prime}, n_{d}\right)}\left(n^{\prime}, n_{d}\right) \cap \mathbb{Z}^{d}}\left|D_{d} g_{i, \mu}(\vec{k})\right|\right)^{1-\beta} \\
& \times\left(\sum_{\substack{\left|n^{\prime}\right| \geq 2 \Lambda \\
n^{\prime} \in \mathbb{Z}^{d-1}}} \sum_{n_{d} \in Y_{n^{\prime}}^{+}}\left(F\left(r\left(n^{\prime}, n_{d}\right)\right)^{-\frac{q(m d-\alpha+\beta)}{d}} \sum_{\vec{k} \in B_{(\lambda+1) r\left(n^{\prime}, n_{d}\right)}\left(n^{\prime}, n_{d}\right) \cap \mathbb{Z}^{d}} g_{i, \mu}(\vec{k})\right)^{\beta} .\right.
\end{aligned}
$$

Note that

$$
\begin{aligned}
& \sum_{\substack{\left|n^{\prime}\right| \geq 2 \Lambda \\
n^{\prime} \in \mathbb{Z}^{d-1}}} \sum_{n_{d} \in Y_{n^{\prime}}^{+}}\left(F\left(r\left(n^{\prime}, n_{d}\right)\right)^{-\frac{q(m d-\alpha+\beta)}{d}} \sum_{\vec{k} \in B_{(\lambda+1) r\left(n^{\prime}, n_{d}\right)}\left(n^{\prime}, n_{d}\right) \cap \mathbb{Z}^{d}}\left|D_{d} g_{i, \mu}(\vec{k})\right|\right. \\
& =\sum_{\vec{k} \in \mathbb{Z}^{d}}\left|D_{d} g_{i, \mu}(\vec{k})\right| \sum_{\substack{\left|n^{\prime}\right| \geq 2 \Lambda \\
n^{\prime} \in \mathbb{Z}^{d-1}}} \sum_{n_{d} \in Y_{n^{\prime}}^{+}}\left(F\left(r\left(n^{\prime}, n_{d}\right)\right)^{-\frac{q(m d-\alpha+\beta)}{d}}\right. \\
& \quad \times \chi_{\left\{\left|\vec{k}-\left(n^{\prime}, n_{d}\right)\right| \leq(\lambda+1) r\left(n^{\prime}, n_{d}\right)\right\}} .
\end{aligned}
$$

Fix $\vec{k}=\left(k^{\prime}, k_{d}\right)$. When $\left|k^{\prime}\right| \geq \Lambda$, we get from (36) that

$$
\sum_{\substack{\left|n^{\prime}\right| \geq 2 \Lambda \\ n^{\prime} \in \mathbb{Z}^{d-1}}} \sum_{n_{d} \in Y_{n^{\prime}}^{+}}\left(F\left(r\left(n^{\prime}, n_{d}\right)\right)^{-\frac{q(m d-\alpha+\beta)}{d}} \chi_{\left\{\left|\left(k^{\prime}-n^{\prime}, k_{d}-n_{d}\right)\right| \leq(\lambda+1) r\left(n^{\prime}, n_{d}\right)\right\}} \lesssim_{\alpha, m, d, \beta, q, \lambda} 1 .\right.
$$

When $\left|k^{\prime}\right|<\Lambda$, note that $\left|n^{\prime}-k^{\prime}\right|>\Lambda>8 \sqrt{d}(\lambda+1)$ and $q(m d-\alpha+\beta)>d$. Then by Lemma 2.1 and (41) we have

$$
\begin{aligned}
& \sum_{\substack{\left|n^{\prime}\right| \geq 2 \Lambda \\
n^{\prime} \in \mathbb{Z}^{d-1}}} \sum_{n_{d} \in Y_{n^{\prime}}^{+}}\left(F\left(r\left(n^{\prime}, n_{d}\right)\right)^{-\frac{q(m d-\alpha+\beta)}{d}} \chi_{\left\{\left|\left(\mid k^{\prime}-n^{\prime}, k_{d}-n_{d}\right)\right| \leq(\lambda+1) r\left(n^{\prime}, n_{d}\right)\right\}}\right. \\
& \leq c_{d}^{-q(m d-\alpha+\beta)} \sum_{\substack{\left|n^{\prime}\right| \geq 2 \Lambda \\
n^{\prime} \in \mathbb{Z}^{d-1}}} \sum_{n_{d} \in Y_{n^{\prime}}^{+}}\left(\frac{\left|\left(k^{\prime}-n^{\prime}, k_{d}-n_{d}\right)\right|}{\lambda+1}-\sqrt{d}\right)^{-q(m d-\alpha+\beta)} \\
& \lesssim_{\alpha, m, d, \beta, q, \lambda} \sum_{\substack{|\vec{n}| \geq \Lambda \\
\vec{n} \in \mathbb{Z}^{d}}}|\vec{n}|^{-q(m d-\alpha+\beta)} \lesssim_{\alpha, m, d, \beta, q, \lambda} \Lambda^{d-q(m d-\alpha+\beta)} \lesssim_{\alpha, m, d, \beta, q, \lambda} \epsilon .
\end{aligned}
$$

It follows from (40), (42), and (51)-(53) that

$$
\sum_{\substack{\left|n^{\prime}\right| \geq 2 \Lambda \\ n^{\prime} \in \mathbb{Z}^{d-1}}} \sum_{n_{d} \in Y_{n^{\prime}}^{+}}\left(F\left(r\left(n^{\prime}, n_{d}\right)\right)^{-\frac{q(m d-\alpha+\beta)}{d}} \sum_{\vec{k} \in B_{(\lambda+1) r\left(n^{\prime}, n_{d}\right)}\left(n^{\prime}, n_{d}\right) \cap \mathbb{Z}^{d}}\left|D_{d} g_{i, \mu}(\vec{k})\right|\right.
$$




$$
\begin{aligned}
& \lesssim_{\alpha, \beta, q, m, d, \lambda} \sum_{\substack{\left|k^{\prime}\right| \geq \Lambda \\
k^{\prime} \in \mathbb{Z}^{d-1}}}\left|D_{d} g_{i, \mu}(\vec{k})\right|+\sum_{\substack{\left|k^{\prime}\right|<\Lambda \\
k^{\prime} \in \mathbb{Z}^{d-1}}}\left|D_{d} g_{i, \mu}(\vec{k})\right| \epsilon \\
& \lesssim_{\alpha, m, d, \beta, q, \lambda}\left\|D_{d} g_{i, \mu} \chi_{\left(B_{\Lambda}(\overrightarrow{0})\right)^{c}}\right\|_{\ell^{1}\left(\mathbb{Z}^{d}\right)}+2\left\|g_{i, \mu}\right\|_{\ell^{1}\left(\mathbb{Z}^{d}\right)} \epsilon \\
& \lesssim_{\alpha, m, d, \beta, q, \lambda, \vec{f}} \epsilon .
\end{aligned}
$$

Similarly,

$$
\begin{aligned}
& \sum_{\substack{\left|n^{\prime}\right| \geq 2 \Lambda \\
n^{\prime} \in \mathbb{Z}^{d-1}}} \sum_{n_{d} \in Y_{n^{\prime}}^{+}}\left(F\left(r\left(n^{\prime}, n_{d}\right)\right)^{-\frac{q(m d-\alpha+\beta)}{d}} \sum_{\vec{k} \in B_{(\lambda+1) r\left(n^{\prime}, n_{d}\right)}\left(n^{\prime}, n_{d}\right) \cap \mathbb{Z}^{d}} g_{i, \mu}(\vec{k})\right. \\
& \quad \lesssim_{\alpha, m, d, \beta, q, \lambda, \vec{f}} \in .
\end{aligned}
$$

Then (48) follows from (40), (50), and (54)-(55). It follows from (47)-(49) that

$$
A_{1} \lesssim_{\alpha, m, d, \beta, q, \lambda, \vec{f}} \epsilon .
$$

It remains to estimate $A_{2}$. For each $n^{\prime} \in \mathbb{Z}^{d-1}$, let

$$
\begin{aligned}
& Z_{n^{\prime}}^{+}=\left\{\left|n_{d}\right| \geq 2 \Lambda: \mathrm{M}_{\alpha, \mathcal{B}}^{\lambda}\left(\vec{g}_{i}\right)\left(n^{\prime}, n_{d}+1\right)<\mathrm{M}_{\alpha, \mathcal{B}}^{\lambda}\left(\vec{g}_{i}\right)\left(n^{\prime}, n_{d}\right)\right\}, \\
& Z_{n^{\prime}}^{-}=\left\{\left|n_{d}\right| \geq 2 \Lambda: \mathrm{M}_{\alpha, \mathcal{B}}^{\lambda}\left(\vec{g}_{i}\right)\left(n^{\prime}, n_{d}+1\right)>\mathrm{M}_{\alpha, \mathcal{B}}^{\lambda}\left(\vec{g}_{i}\right)\left(n^{\prime}, n_{d}\right)\right\}, \\
& Z_{n^{\prime}}^{-}=\left\{\left|n_{d}\right| \geq 2 \Lambda: \mathrm{M}_{\alpha, \mathcal{B}}^{\lambda}\left(\vec{g}_{i}\right)\left(n^{\prime}, n_{d}+1\right)=\mathrm{M}_{\alpha, \mathcal{B}}^{\lambda}\left(\vec{g}_{i}\right)\left(n^{\prime}, n_{d}\right)\right\} .
\end{aligned}
$$

Then we have

$$
\begin{aligned}
A_{2} \leq & \sum_{n^{\prime} \in \mathbb{Z}^{d-1}} \sum_{n_{d} \in Z_{n^{\prime}}^{+}}\left(\mathrm{M}_{\alpha, \mathcal{B}}^{\lambda}\left(\vec{g}_{i}\right)\left(n^{\prime}, n_{d}\right)-\mathrm{M}_{\alpha, \mathcal{B}}^{\lambda}\left(\vec{g}_{i}\right)\left(n^{\prime}, n_{d}+1\right)\right)^{q} \\
& +\sum_{n^{\prime} \in \mathbb{Z}^{d-1}} \sum_{n_{d} \in Z_{n^{\prime}}^{-}}\left(\mathrm{M}_{\alpha, \mathcal{B}}^{\lambda}\left(\vec{g}_{i}\right)\left(n^{\prime}, n_{d}+1\right)-\mathrm{M}_{\alpha, \mathcal{B}}^{\lambda}\left(\vec{g}_{i}\right)\left(n^{\prime}, n_{d}\right)\right)^{q} .
\end{aligned}
$$

We want to show that

$$
\begin{aligned}
& \sum_{n^{\prime} \in \mathbb{Z}^{d-1}} \sum_{n_{d} \in Z_{n^{\prime}}^{+}}\left(\mathrm{M}_{\alpha, \mathcal{B}}^{\lambda}\left(\vec{g}_{i}\right)\left(n^{\prime}, n_{d}\right)-\mathrm{M}_{\alpha, \mathcal{B}}^{\lambda}\left(\vec{g}_{i}\right)\left(n^{\prime}, n_{d}+1\right)\right)^{q} \lesssim_{\alpha, m, d, \beta, q, \lambda, \vec{f}} \epsilon \\
& \sum_{n^{\prime} \in \mathbb{Z}^{d-1}} \sum_{n_{d} \in Z_{n^{\prime}}^{-}}\left(\mathrm{M}_{\alpha, \mathcal{B}}^{\lambda}\left(\vec{g}_{i}\right)\left(n^{\prime}, n_{d}+1\right)-\mathrm{M}_{\alpha, \mathcal{B}}^{\lambda}\left(\vec{g}_{i}\right)\left(n^{\prime}, n_{d}\right)\right)^{q} \lesssim_{\alpha, m, d, \beta, q, \lambda, \vec{f}} \epsilon .
\end{aligned}
$$

We only prove (58), and (59) is analogous. Since all $g_{i, j} \in \ell^{1}\left(\mathbb{Z}^{d}\right)$, then for any $\left(n^{\prime}, n_{d}\right) \in \mathbb{Z}^{d}$ with $n_{d} \in Z_{n^{\prime}}^{+}$, there exist $\vec{x} \in \mathbb{R}^{d}$ and $r\left(n^{\prime}, n_{d}\right)>0$ such that $\left|\left(n^{\prime}, n_{d}\right)-\vec{x}\right| \leq \lambda r\left(n^{\prime}, n_{d}\right)$ and $\mathrm{M}_{\alpha, \mathcal{B}}^{\lambda}\left(\vec{g}_{i}\right)\left(n^{\prime}, n_{d}\right)=\mathrm{A}_{r\left(n^{\prime}, n_{d}\right)}\left(\vec{g}_{i}\right)(\vec{x})$. By the arguments similar to those used to derived (32),

$$
\begin{aligned}
& \sum_{n^{\prime} \in \mathbb{Z}^{d-1}} \sum_{n_{d} \in Z_{n^{\prime}}^{+}}\left(\mathrm{M}_{\alpha, \mathcal{B}}^{\alpha}\left(\vec{g}_{i}\left(n^{\prime}, n_{d}\right)-\mathrm{M}_{\alpha, \mathcal{B}}^{\alpha}\left(\vec{g}_{i}\right)\left(n^{\prime}, n_{d}+1\right)\right)^{q}\right. \\
& \quad \sum_{m, d} \sum_{\mu=1}^{m}\left(\prod_{1 \leq j \neq \mu \leq m}\left\|g_{i, j}\right\|_{\ell^{1}\left(\mathbb{Z}^{d}\right)}^{q}\right)\left(\left\|D_{d} g_{i, \mu}\right\|_{\ell^{1}\left(\mathbb{Z}^{d}\right)}^{(q-1)(1-\beta)}\left\|g_{i, \mu}\right\|_{\ell^{1}\left(\mathbb{Z}^{d}\right)}^{(q-1) \beta}\right)
\end{aligned}
$$




$$
\begin{aligned}
& \times\left(\sum _ { n ^ { \prime } \in \mathbb { Z } ^ { d - 1 } } \sum _ { n _ { d } \in Z _ { n ^ { \prime } } ^ { + } } \left(F\left(r\left(n^{\prime}, n_{d}\right)\right)^{-\frac{q(m d-\alpha+\beta)}{d}}\right.\right. \\
& \left.\times \sum_{\vec{k} \in B_{(\lambda+1) r\left(n^{\prime}, n_{d}\right)}\left(n^{\prime}, n_{d}\right) \cap \mathbb{Z}^{d}}\left|D_{d} g_{i, \mu}(\vec{k})\right|\right)^{1-\beta} \\
& \times\left(\sum_{n^{\prime} \in \mathbb{Z}^{d-1}} \sum_{n_{d} \in Z_{n^{\prime}}^{+}}\left(F\left(r\left(n^{\prime}, n_{d}\right)\right)^{-\frac{q(m d-\alpha+\beta)}{d}} \sum_{\vec{k} \in B_{(\lambda+1) r\left(n^{\prime}, n_{d}\right)}\left(n^{\prime}, n_{d}\right) \cap \mathbb{Z}^{d}} g_{i, \mu}(\vec{k})\right)^{\beta} .\right.
\end{aligned}
$$

Note that

$$
\begin{aligned}
& \sum_{n^{\prime} \in \mathbb{Z}^{d-1}} \sum_{n_{d} \in Z_{n^{\prime}}^{+}}\left(F\left(r\left(n^{\prime}, n_{d}\right)\right)^{-\frac{q(m d-\alpha+\beta)}{d}} \sum_{\vec{k} \in B_{(\lambda+1)\left(n^{\prime}, n_{d}\right)}\left(n^{\prime}, n_{d}\right) \cap \mathbb{Z}^{d}}\left|D_{d} g_{i, \mu}(\vec{k})\right|\right. \\
& =\sum_{\vec{k} \in \mathbb{Z}^{d}}\left|D_{d} g_{i, \mu}(\vec{k})\right| \sum_{\substack { n^{\prime} \in \mathbb{Z}^{d-1}-1 \\
\begin{subarray}{c}{\left|n_{d}\right| \geq 2 \Lambda \\
n_{d} \in \mathbb{Z}{ n ^ { \prime } \in \mathbb { Z } ^ { d - 1 } - 1 \\
\begin{subarray} { c } { | n _ { d } | \geq 2 \Lambda \\
n _ { d } \in \mathbb { Z } } }\end{subarray}}\left(F\left(r\left(n^{\prime}, n_{d}\right)\right)^{-\frac{q(m d-\alpha+\beta)}{d}}\right. \\
& \quad \times \chi_{\left\{\left|\vec{k}-\left(n^{\prime}, n_{d}\right)\right| \leq(\lambda+1) r\left(n^{\prime}, n_{d}\right)\right\}} .
\end{aligned}
$$

Fix $\vec{k}=\left(k^{\prime}, k_{d}\right)$. When $\left|k_{d}\right|<\Lambda$, note that $\left|n_{d}-k_{d}\right|>\Lambda>8 \sqrt{d}(\lambda+1)$ and $q(m d-\alpha+\beta)>d$. Invoking Lemma 2.1, we have

$$
\begin{aligned}
& \sum_{n^{\prime} \in \mathbb{Z}^{d-1}} \sum_{\substack{\left|n_{d}\right| \geq 2 \Lambda \\
n_{d} \in \mathbb{Z}}}\left(F\left(r\left(n^{\prime}, n_{d}\right)\right)^{-\frac{q(m d-\alpha+\beta)}{d}} \chi_{\left\{\left|\vec{k}-\left(n^{\prime}, n_{d}\right)\right| \leq(\lambda+1) r\left(n^{\prime}, n_{d}\right)\right\}}\right. \\
& =\sum_{n^{\prime} \in \mathbb{Z}^{d-1}} \sum_{\substack{\left|n_{d}\right| \geq 2 \Lambda \\
n_{d} \in \mathbb{Z}}}\left(F\left(r\left(n^{\prime}, n_{d}\right)\right)^{-\frac{q(m d-\alpha+\beta)}{d}} \chi_{\left\{4 \sqrt{d}(\lambda+1) \leq\left|\vec{k}-\left(n^{\prime}, n_{d}\right)\right| \leq(\lambda+1) r\left(n^{\prime}, n_{d}\right)\right\}}\right. \\
& \leq c_{d}^{-\frac{q(m d-\alpha+\beta)}{d}} \sum_{n^{\prime} \in \mathbb{Z}^{d-1}} \sum_{\substack{\left|n_{d}\right| \geq 2 \Lambda \\
n_{d} \in \mathbb{Z}}}\left(\frac{\left|\left(k^{\prime}-n^{\prime}, k_{d}-n_{d}\right)\right|}{\lambda+1}-\sqrt{d}\right)^{-q(m d-\alpha+\beta)} \\
& \lesssim_{\alpha, m, d, \beta, q, \lambda} \sum_{\substack{|\vec{n}| \geq \Lambda \\
\vec{n} \in \mathbb{Z}^{d}}}|\vec{n}|^{-q(m d-\alpha+\beta)} \lesssim_{\alpha, m, d, \beta, q, \lambda} \Lambda^{d-q(m d-\alpha+\beta)} \lesssim_{\alpha, m, d, \beta, q, \lambda} \epsilon .
\end{aligned}
$$

When $\left|k_{d}\right| \geq \Lambda$, we get easily from (36) that

$$
\sum_{n^{\prime} \in \mathbb{Z}^{d-1}} \sum_{\substack{\left|n_{d}\right| \geq 2 \Lambda \\ n_{d} \in \mathbb{Z}}}\left(F\left(r\left(n^{\prime}, n_{d}\right)\right)^{-\frac{q(m d-\alpha+\beta)}{d}} \chi_{\left\{\left|\vec{k}-\left(n^{\prime}, n_{d}\right)\right| \leq(\lambda+1) r\left(n^{\prime}, n_{d}\right)\right\}} \lesssim \alpha, m, d, \beta, q, \lambda 1 .\right.
$$

It follows from (40) and (61)-(63) that

$$
\begin{aligned}
& \sum_{n^{\prime} \in \mathbb{Z}^{d-1}} \sum_{n_{d} \in Z_{n^{\prime}}^{+}}\left(F\left(r\left(n^{\prime}, n_{d}\right)\right)^{-\frac{q(m d-\alpha+\beta)}{d}} \sum_{\vec{k} \in B_{(\lambda+1) r\left(n^{\prime}, n_{d}\right)}\left(n^{\prime}, n_{d}\right) \cap \mathbb{Z}^{d}}\left|D_{d} g_{i, \mu}(\vec{k})\right|\right. \\
& \lesssim_{\alpha, m, d, \beta, q, \lambda, \vec{f}} \epsilon .
\end{aligned}
$$


Similarly,

$$
\begin{aligned}
& \sum_{n^{\prime} \in \mathbb{Z}^{d-1}} \sum_{n_{d} \in Z_{n^{\prime}}^{+}}\left(F\left(r\left(n^{\prime}, n_{d}\right)\right)^{-\frac{q(m d-\alpha+\beta)}{d}} \sum_{\vec{k} \in B_{(\lambda+1) r\left(n^{\prime}, n_{d}\right)}\left(n^{\prime}, n_{d}\right) \cap \mathbb{Z}^{d}} g_{i, d}(\vec{k})\right. \\
& \lesssim_{\alpha, m, d, \beta, q, \lambda, \vec{f}} \epsilon .
\end{aligned}
$$

Combining (60) with (40) and (64)-(65) yields (58). We get from (57)-(59) that

$$
A_{2} \lesssim_{\alpha, m, d, \beta, q, \lambda, \vec{f}} \epsilon
$$

It follows from (45)-(46), (56), and (66) that

$$
\left\|D_{l} \mathrm{M}_{\alpha, \mathcal{B}}^{\lambda}\left(\vec{g}_{i}\right)-D_{l} \mathrm{M}_{\alpha, \mathcal{B}}^{\lambda}(\vec{f})\right\|_{\ell^{q}\left(\mathbb{Z}^{d}\right)} \lesssim_{\alpha, m, d, \beta, q, \lambda, \vec{f}} \epsilon \quad \forall i \geq \max \left\{N_{1}, N_{2}\right\}
$$

This yields (39) for $l=d$.

\subsection{Proof of Theorem 1.5 for $M_{\alpha, \mathcal{R}}^{\lambda}$}

The proof of Theorem 1.5 for $\mathrm{M}_{\alpha, \mathcal{R}}^{\lambda}$ is similar as for $\mathrm{M}_{\alpha, \mathcal{B}}^{\lambda}$. We only replace the norm | | | with $\|\cdot\|_{\infty}$. The details are left to the interested reader.

\section{Acknowledgements}

The author thanks anonymous referees for their valuable suggestions.

\section{Funding}

This work was supported by the Natural Science Foundation of University Union of Science and Technology Department of Fujian Province (No. 2019J01784), the Youth Foundation of Fujian Province (Grant No. JAT170398), and the Natural Science Foundation of Fujian University of Technology (Nos. GY-Z15124, GY-Z160129).

\section{Availability of data and materials}

Not applicable.

\section{Competing interests}

The author declares that there is no conflict of interests.

\section{Authors' contributions}

The author read and approved the final manuscript.

\section{Publisher's Note}

Springer Nature remains neutral with regard to jurisdictional claims in published maps and institutional affiliations.

Received: 17 April 2019 Accepted: 25 July 2019 Published online: 23 September 2019

\section{References}

1. Aldaz, J.M., Pérez Lázaro, J.: Functions of bounded variation, the derivative of the one dimensional maximal function, and applications to inequalities. Trans. Am. Math. Soc. 359(5), 2443-2461 (2007)

2. Bober, J., Carneiro, E., Hughes, K., Pierce, L.B.: On a discrete version of Tanaka's theorem for maximal functions. Proc. Am. Math. Soc. 140(5), 1669-1680 (2012)

3. Brezis, H., Lieb, E.: A relation between pointwise convergence of functions and convergence of functionals. Proc. Am. Math. Soc. 88, 486-490 (1983)

4. Carneiro, E., Finder, R., Sousa, M.: On the variation of maximal operators of convolution type II. Rev. Mat. Iberoam. (2019, to appear)

5. Carneiro, E., Hughes, K.: On the endpoint regularity of discrete maximal operators. Math. Res. Lett. 19(6), 1245-1262 (2012)

6. Carneiro, E., Madrid, J., Pierce, L.B.: Endpoint Sobolev and BV continuity for maximal operators. arXiv:1708.06051v1

7. Carneiro, E., Mardid, J.: Derivative bounds for fractional maximal functions. Trans. Am. Math. Soc. 369, 4063-4092 (2017)

8. Carneiro, E., Moreira, D.: On the regularity of maximal operators. Proc. Am. Math. Soc. 136(12), 4395-4404 (2008) 
9. Carneiro, E., Svaiter, B.F.: On the variation of maximal operators of convolution type. J. Funct. Anal. 265, 837-865 (2013)

10. Hajłasz, P., Maly, J.: On approximate differentiability of the maximal function. Proc. Am. Math. Soc. 138, 165-174 (2010)

11. Hajłasz, P., Onninen, J.: On boundedness of maximal functions in Sobolev spaces. Ann. Acad. Sci. Fenn., Math. 29(1), 167-176 (2004)

12. Kinnunen, J.: The Hardy-Littlewood maximal function of a Sobolev function. Isr. J. Math. 100, 117-124 (1997)

13. Kinnunen, J., Lindqvist, P.: The derivative of the maximal function. J. Reine Angew. Math. 503, 161-167 (1998)

14. Kinnunen, J., Saksman, E.: Regularity of the fractional maximal function. Bull. Lond. Math. Soc. 35(4), $529-535$ (2003)

15. Korry, S.: Boundedness of Hardy-Littlewood maximal operator in the framework of Lizorkin-Triebel spaces. Rev. Mat. Complut. 15(2), 401-416(2002)

16. Korry, S.: A class of bounded operators on Sobolev spaces. Arch. Math. 82(1), 40-50 (2004)

17. Kurka, O.: On the variation of the Hardy-Littlewood maximal function. Ann. Acad. Sci. Fenn., Math. 40, 109-133 (2015)

18. Liu, F.: A remark on the regularity of the discrete maximal operator. Bull. Aust. Math. Soc. 95, 108-120 (2017)

19. Liu, F:: On the regularity of one-sided fractional maximal functions. Math. Slovaca 68(5), 1097-1112 (2018)

20. Liu, F.: Continuity and approximate differentiability of multisublinear fractional maximal functions. Math. Inequal. Appl. 21(1), 25-40 (2018)

21. Liu, F.: A note on Marcinkiewicz integrals associated to surfaces of revolution. J. Aust. Math. Soc. 104, 380-402 (2018)

22. Liu, F.: Boundedness and continuity of maximal operators associated to polynomial compound curves on Triebel-Lizorkin spaces. Math. Inequal. Appl. 22(1), 25-44 (2019)

23. Liu, F., Chen, T., Wu, H.: A note on the endpoint regularity of the Hardy-Littlewood maximal functions. Bull. Aust. Math. Soc. 94, 121-130 (2016)

24. Liu, F., Mao, S.: On the regularity of the one-sided Hardy-Littlewood maximal functions. Czechoslov. Math. J. 67(142), 219-234 (2017)

25. Liu, F., Wu, H.: On the regularity of the multisublinear maximal functions. Can. Math. Bull. 58(4), 808-817 (2015)

26. Liu, F., Wu, H.: Endpoint regularity of multisublinear fractional maximal functions. Can. Math. Bull. 60(3), 586-603 (2017)

27. Liu, F., Wu, H.: Regularity of discrete multisublinear fractional maximal functions. Sci. China Math. 60(8), 1461-1476 (2017)

28. Liu, F., Wu, H.: On the regularity of maximal operators supported by submanifolds. J. Math. Anal. Appl. 453, 144-158 (2017)

29. Liu, F., Wu, H.: A note on the endpoint regularity of the discrete maximal operator. Proc. Am. Math. Soc. 147(2), 583-596 (2019)

30. Luiro, H.: Continuity of the maximal operator in Sobolev spaces. Proc. Am. Math. Soc. 135(1), 243-251 (2007)

31. Luiro, H.: On the regularity of the Hardy-Littlewood maximal operator on subdomains of $\mathbb{R}^{d}$. Proc. Edinb. Math. Soc. 53(1), 211-237 (2010)

32. Madrid, J.: Sharp inequalities for the variation of the discrete maximal function. Bull. Aust. Math. Soc. 95, 94-107 (2017)

33. Miao, A., Zhang, T., Zhang, J., Wang, C.: Dynamics of a stochastic SIR model with both horizontal and vertical transmission. J. Appl. Anal. Comput. 8(4), 1108-1121 (2018)

34. Pierce, L.B.: Discrete Analogues in Harmonic Analysis. Ph.D. Thesis, Princeton University (2009)

35. Tanaka, $\mathrm{H}$ :: A remark on the derivative of the one-dimensional Hardy-Littlewood maximal function. Bull. Aust. Math. Soc. 65(2), 253-258 (2002)

36. Temur, F.: On regularity of the discrete Hardy-Littlewood maximal function. arXiv:1303.3993

37. Zhu, J., Hao, B.: A new smoothing method for solving nonlinear complementarity problems. Open Math. 17, 104-119 (2019)

\section{Submit your manuscript to a SpringerOpen ${ }^{\circ}$ journal and benefit from:}

- Convenient online submission

- Rigorous peer review

- Open access: articles freely available online

- High visibility within the field

- Retaining the copyright to your article

Submit your next manuscript at springeropen.com 